\title{
Top-down and Bottom-up Regulated Auditory Phantom Perception
}

\author{
Sven Vanneste, ${ }^{1}$ Ola Alsalman, ${ }^{1}$ and Dirk De Ridder ${ }^{2}$ \\ ${ }^{1}$ Laboratory for Clinical and Integrative Neuroscience, School of Behavioral and Brain Sciences, The University of Texas at Dallas, Richardson, Texas 75080 , \\ and ${ }^{2}$ Department of Surgical Sciences, Dunedin School of Medicine, University of Otago, Dunedin 9054, New Zealand
}

Auditory phantom percepts such as tinnitus are associated with auditory deafferentation. The idea is that auditory deafferentation limits the amount of information the brain can acquire to make sense of the world. Because of this, auditory deafferentation increases the uncertainty of the auditory environment. To minimize uncertainty, the deafferented brain will attempt to obtain or fill in the missing information. A proposed multiphase compensation model suggests two distinct types of bottom-up related tinnitus: an auditory cortex related tinnitus and a parahippocampal cortex related tinnitus. The weakness of this model is that it cannot explain why some people without hearing loss develop tinnitus, whereas conversely others with hearing loss do not develop tinnitus. In this human study, we provide evidence for a top-down type of tinnitus associated with a deficient noise-cancelling mechanism. A total of 72 participants (age: $40.96 \pm 7.67$ years; males: 48 ; females: 24 ) were recruited for this study. We demonstrate that top-down related tinnitus is related to a change in the pregenual anterior cingulate cortex that corresponds to increased activity in the auditory cortex. This is in accordance with the idea that tinnitus can have different generators as proposed in a recent model that suggests that different compensation mechanisms at a cortical level can be linked to phantom percepts.

Key words: auditory cortex; hearing loss; parahippocampus; pregenual anterior cingulate cortex; tinnitus; top-down

\section{Significance Statement}

Chronic tinnitus affects $15 \%$ of the population worldwide. The term "tinnitus" however represents a highly heterogeneous condition, as evidenced by the fact that there are no effective treatments or even an adequate understanding of the underlying neural mechanisms. Consistent with this idea, our research shows that tinnitus indeed has different subtypes related to hearing loss. In a human study tightly controlled for hearing loss, we establish a tinnitus subtype associated with a deficient top-down noisecancelling mechanism, which distinguishes it from bottom-up subtypes. We demonstrate that top-down related tinnitus relates to a change in the pregenual anterior cingulate cortex that corresponds to increased activity in the auditory cortex, whereas bottom-up tinnitus instead relates to changes in the parahippocampal cortex.

\section{Introduction}

Tinnitus, the phantom perception of a sound in the absence of an external stimulus, is a disorder that affects $15 \%$ of the population worldwide (Axelsson and Ringdahl, 1989). Typically, tinnitus has been associated with auditory deafferentation (e.g., noise trauma, presbyacusis, etc.; Vanneste and De Ridder, 2016). A theoretical multiphase compensation mechanism at a cortical level has been

\footnotetext{
Received April 16, 2018; revised 0ct. 18, 2018; accepted 0ct. 20, 2018.

Author contributions: S.V. and 0.A. wrote the first draft of the paper; S.V. and D.D.R. edited the paper; S.V. and $0 . A$. designed research; $0 . A$. performed research; $0 . A$. contributed unpublished reagents/analytic tools; S.V. and O.A. analyzed data; S.V. and D.D.R. wrote the paper.

The authors declare no competing financial interests.

Correspondence should be addressed to Sven Vanneste, Lab for Clinical and Integrative Neuroscience, School of

Behavioral and Brain Science, University of Texas at Dallas, 800 West Campbell Road, Richardson, Texas 75080. E-mail: sven.vanneste@utdallas.edu.

https://doi.org/10.1523/JNEUROSCI.0966-18.2018

Copyright $\odot 2019$ the authors $\quad 0270-6474 / 19 / 390364-15 \$ 15.00 / 0$
}

hypothesized that links bottom-up auditory deafferentation to tinnitus (De Ridder et al., 2014a). The idea is that auditory deafferentation limits the amount of information the brain can acquire to make sense of the world. Because of this, auditory deafferentation increases the uncertainty of the auditory environment. To minimize uncertainty, the deafferented brain will attempt to obtain or fill in the missing information (De Ridder et al., 2014a).

The multiphase compensation mechanism model hypothesizes that the tinnitus percept depends on the amount of hearing loss or deafferented auditory channels (De Ridder et al., 2015b). This hypothesis was supported in a recent study revealing that two different kinds of tinnitus can be discerned depending on the presence of hearing loss: an auditory cortex related tinnitus associated with slight to mild hearing loss and a parahippocampal cortex related tinnitus associated with significant hearing loss (Vanneste and De Ridder, 2016). In mild hearing loss subjects, 
the missing information can be retrieved from the auditory cortical neighborhood, decreasing surround inhibition, characterized by a slowing down of resting state alpha to theta activity associated with an increase in surrounding gamma activity (Weisz et al., 2005a, 2007a). When hearing loss is more severe, the missing information is retrieved from theta-gamma-mediated parahippocampal auditory memory (Vanneste and De Ridder, 2016).

The weakness of the multiphase compensation model is that it cannot explain why some people with normal hearing develop tinnitus, while conversely others with hearing loss do not develop tinnitus. Interestingly, recent research suggests that a top-down modulatory system for an auditory percept could play a role (Rauschecker et al., 2015). The idea is that the pregenual anterior cingulate cortex oscillates at theta frequencies, but that an activated functioning top-down noise-cancelling mechanism works predominantly in the alpha frequency band during the resting state (De Ridder et al., 2015b). Given a dysfunctional noisecancelling mechanism, however, it is expected that theta and gamma would predominate (De Ridder et al., 2015b). Preliminary evidence of this was obtained in a study looking at selective enriched acoustic stimulation, in which an overcompensation for hearing loss was used (Vanneste et al., 2013). Clinical worsening of patients was related to increased theta and gamma activity in the pregenual anterior cingulate cortex, linked to increased gamma in the auditory cortex (Vanneste et al., 2013). In contrast, using bifrontal transcranial direct current stimulation suppresses the tinnitus percept in association with a decrease in theta and gamma band activity in the auditory cortex (De Ridder et al., $2015 b$ ), associated with via increased alpha activity in the pregenual anterior cingulate cortex (Vanneste and De Ridder, 2011).

Even though evidence provides support for a top-down related tinnitus related to a dysfunctional noise-cancelling mechanism, these findings have still to be considered as preliminary as these studies were not well controlled for hearing loss or are not replicated by our labs. It has been proposed that an auditory phantom phenomenon can be either associated with bottom-up deafferentation, a deficient top-down noise-cancelling mechanism, or a combination of both (De Ridder et al., 2014b). For participants with hearing loss but no tinnitus, we hypothesize that a top-down modulatory system suppresses the phantom percept as reflected by increased activity in the pregenual anterior cingulate cortex within the alpha frequency band. For tinnitus patients without hearing loss, we hypothesize that this noisecancelling mechanism is deficient, oscillating at non-activated resting state theta frequencies that is coupled with increased gamma activity in the pregenual anterior cingulate cortex linked to increased gamma in the auditory cortex. On the other hand, patients with hearing loss will show increased activity in the auditory and parahippocampal cortex for the theta and gamma frequency band. To test these hypotheses, we will collect electrophysiological data (i.e., electroencephalography) and assess activity, connectivity (both functional and effective), and phaseamplitude coupling changes between participants with and without tinnitus that have or do not have hearing loss.

\section{Materials and Methods}

\section{Subjects}

A total of 72 participants (age: $40.96 \pm 7.67$ years; males: 48 ; females: 24 ) were recruited for this study based on their age, gender, audiometry, and whether or not they perceived tinnitus. All patients were screened for the extent of hearing loss ( $\mathrm{dB} \mathrm{HL}$ ) using pure tone audiometry per the British Society of Audiology procedures at $0.125,0.25,0.5,1,2,3,4,6$, and $8 \mathrm{kHz}$
(British Society of Audiology, 2008). Individuals with pulsatile tinnitus; Ménière's disease; otosclerosis; chronic headache; neurological disorders such as brain tumors, traumatic brain injury, or stroke; and individuals being treated for mental disorders were not included in the study to maximize the sample homogeneity. The study was in accordance with the ethical standards of the Helsinki declaration (1964) and was approved by the Institutional Review Board of the University of Texas at Dallas (1506). All participants gave their written, informed consent per the approved guidelines.

Thirty-six participants perceived tinnitus, whereas 36 participants reportedly did not. Of the 36 tinnitus patients, 18 showed normal hearing based on the pure tone audiogram, whereas for the other 18 participants the audiogram showed mild hearing loss. For the 36 participants who did not perceive tinnitus, 18 reported normal hearing based on the pure tone audiogram, whereas for the other 18 participants the audiogram showed mild hearing loss. Based on the hearing loss classification introduced by Clark (1981), both the tinnitus subjects and no-tinnitus subjects that have an overall hearing loss (overall frequencies) of $5.31(\mathrm{SD}=1.32)$ and $4.79 \mathrm{~dB}$ HL ( $\mathrm{SD}=1.41$ ), were classified as normal hearing groups. Both the tinnitus subject and no-tinnitus subjects that were classified as hearing loss had an overall hearing loss (i.e., over all assessed frequencies) of $26.26 \mathrm{~dB}$ HL (SD = 10.50; range 10-60) and $27.76 \mathrm{~dB} \mathrm{HL}(\mathrm{SD}=10.66$; range 10-65), respectively. Overall this could be classified as mild hearing loss. Based on the hearing loss range, we can classify these latter groups to have slight to moderately severe hearing loss. Throughout this paper, we will refer to these groups as subjects with hearing loss.

All tinnitus participants were interviewed as to the perceived location of the tinnitus (the left ear, in both ears, the right ear) as well as the tinnitus sound characteristics (pure tone-like tinnitus or noise-like tinnitus). Tinnitus patients were further tested for the tinnitus pitch (frequency) by performing a tinnitus matching analysis. In unilateral tinnitus patients, tinnitus matching was performed contralaterally to the tinnitus ear. In bilateral tinnitus patients, tinnitus matching was performed contralaterally to the worse tinnitus ear. First, a $1 \mathrm{kHz}$ pure tone was presented contralaterally to the (worse) tinnitus ear at $10 \mathrm{~dB}$ above the patient's hearing threshold in that ear. The pitch was adjusted until the patient judged the sound to resemble his/her tinnitus most (Meeus et al., 2010, 2011). Based on the tinnitus frequency, we calculated the hearing loss at the tinnitus frequency as obtained by tinnitus matching. For unilateral tinnitus, the hearing loss contralateral to where the patient perceived the tinnitus was considered, whereas for bilateral tinnitus patients we calculated the mean of hearing thresholds. A numeric rating scale for loudness ("How loud is your tinnitus?": $0=$ no tinnitus and $10=$ as loud as imaginable) was assessed to measure the subjective loudness. Also the Tinnitus Questionnaire (TQ) was assessed (Hiller and Goebel, 1992; Hiller et al., 1994). This scale is comprised of 52 items and is a well established measure for the assessment of a broad spectrum of tinnitus-related psychological complaints. The TQ measures emotional and cognitive distress, intrusiveness, auditory perceptual difficulties, sleep disturbances, and somatic complaints. As previously mentioned, the global TQ score can be computed to measure the general level of psychological and psychosomatic distress. In several studies, this measure has been shown to be a reliable and valid instrument in different countries (Hiller and Goebel, 1992; McCombe et al., 2001). A three-point scale is given for all items, ranging from "true" ( 2 points) to "partly true" ( 1 point) and "not true" ( 0 points). The total score (from 0 to 84 ) was computed according to standard criteria published in previous work (Hiller and Goebel, 1992; Hiller et al., 1994). See Table 1 for an overview of the tinnitus characteristics. We also assessed patients using the Beck Depression Inventory (BDI-II), a questionnaire that provides information about depressive mood state (Richter et al., 1998).

As Table 1 demonstrates, no significant differences were found between the four groups (no tinnitus/normal hearing; no tinnitus/hearing loss; tinnitus/normal hearing; tinnitus/hearing loss) for age, gender, BDI, tinnitus duration, tinnitus type, lateralization, tinnitus loudness [numeric rating scale (NRS)], tinnitus distress (TQ), tinnitus frequency $(\mathrm{Hz})$, and tinnitus loudness $(\mathrm{dB})$. A possible concern could be the gender mismatch within each group, however, research has shown that there is no association between gender and tinnitus (Pinto et al., 2010). Gender 
Table 1. Demographics

\begin{tabular}{|c|c|c|c|c|c|}
\hline & \multicolumn{2}{|l|}{ Non-tinnitus } & \multicolumn{2}{|l|}{ Tinnitus } & \multirow[b]{2}{*}{$P$} \\
\hline & $\begin{array}{l}\text { Normal } \\
\text { hearing }\end{array}$ & $\begin{array}{l}\text { Hearing } \\
\text { loss }\end{array}$ & $\begin{array}{l}\text { Normal } \\
\text { hearing }\end{array}$ & $\begin{array}{l}\text { Hearing } \\
\text { loss }\end{array}$ & \\
\hline Age & $41.50(7.56)$ & $41.22(8.90)$ & $40.00(8.01)$ & $41.11(6.61)$ & 0.71 \\
\hline Gender & $\mathrm{M}: 12 / \mathrm{F}: 6$ & $\mathrm{M}: 14 / \mathrm{F}: 4$ & $\mathrm{M}: 10 / \mathrm{F}: 8$ & $\mathrm{M}: 12 / \mathrm{F}: 6$ & 0.45 \\
\hline $\mathrm{BDI}$ & $4.28(2.91)$ & $4.72(2.67)$ & $5.61(1.91)$ & $6.06(2.50)$ & 0.87 \\
\hline Tinnitus duration & & & $3.89(2.00)$ & $4.33(1.84)$ & 0.49 \\
\hline Tinnitus type & & & NBN: 9/PT: 9 & NBN: 12/PT: 6 & 0.50 \\
\hline Tinnitus lateralization & & & UNI: 9/BIL: 9 & UNI: 7/BIL: 11 & 0.74 \\
\hline $\begin{array}{l}\text { Subjective tinnitus } \\
\text { loudness, NRS }\end{array}$ & & & $5.00(2.14)$ & $5.58(2.85)$ & 0.49 \\
\hline Tinnitus distress, TQ & & & 41.89 (13.42) & $49.56(14.18)$ & 0.11 \\
\hline Tinnitus frequency, $\mathrm{Hz}$ & & & 4486.11 (2797.68) & $5180.56(3026.60)$ & 0.48 \\
\hline Tinnitus loudness, dB & & & $9.56(6.09)$ & $11.61(7.33)$ & 0.37 \\
\hline
\end{tabular}

M, Male; F, female; NBN, narrow band noise; PT, pure tone; UNI, unilateral; BIL, bilateral. Hearing loss is measured based on a pure tone audiogram.

differences regarding tinnitus-related distress in patients with chronic tinnitus was shown; however, these differences depended mainly on age and in part on duration of tinnitus (Seydel et al., 2013). However, our data do not show a significant difference between males and females for distress $(F=1.77, p=0.19)$, tinnitus duration $(F=0.40, p=0.53)$, or tinnitus loudness $(F=0.44, p=0.51)$. Figure 1 shows the audiogram for the different groups (no tinnitus/normal hearing; no tinnitus/hearing loss; tinnitus/normal hearing; tinnitus/hearing loss). Again no difference was obtained between hearing loss between males and females $(F=0.38$, $p=0.54)$.

Previous research by our group further showed differences between unilateral and bilateral tinnitus in patients with similar hearing levels (Vanneste et al., 2011). However, a comparison between unilateral and bilateral tinnitus in this study did not show significant changes for each tinnitus group separately (i.e., normal hearing and hearing loss). These could because of a sample size compared with the original study.

\section{EEG collection and processing}

Data collection. Electroencephalography (EEG) data (Neuroscan, http:// compumedicsneuroscan.com/) were obtained in a quiet room while each participant was sitting upright on a comfortable chair. The scalp was cleaned with alcohol wipes before the baseline EEG recording. The EEG was sampled with 64 electrodes in the standard 10-10 International placement and impedances were checked to remain $<5 \mathrm{k} \Omega$. Data were collected eyes-closed (sampling rate $=1 \mathrm{kHz}$, bandpassed DC, $200 \mathrm{~Hz}$ ) and lasted $\sim 5 \mathrm{~min}$. The midline reference was located at the vertex and the ground electrode was located at AFZ. Participants were instructed not to drink alcohol $24 \mathrm{~h}$ before EEG recording or caffeinated beverages $1 \mathrm{~h}$ before recording to avoid alcohol- or caffeine-induced changes in the EEG stream (Volkow et al., 2000; Logan et al., 2002; Siepmann and Kirch, 2002). The alertness of participants was checked by monitoring both slowing of the alpha rhythm and appearance of spindles in the EEG stream to prevent possible enhancement of the theta power because of drowsiness during recording (Moazami-Goudarzi et al., 2010). Off-line data were resampled to $128 \mathrm{~Hz}$, bandpass filtered in the range $2-44 \mathrm{~Hz}$ and subsequently transposed into Eureka! software (Congedo, 2002), plotted, and carefully inspected for manual artifact-rejection. All episodic artifacts including eye blinks, eye movements, teeth clenching, body movement, or ECG artifact were removed from the stream of the EEG. Average Fourier cross-spectral matrices were computed for frequency bands delta $(2-3.5 \mathrm{~Hz})$, theta $(4-7.5 \mathrm{~Hz})$, alpha $(8-12 \mathrm{~Hz})$, beta $(13-30 \mathrm{~Hz})$, and gamma $(30.5-44 \mathrm{~Hz})$.

Source localization. Standardized low-resolution brain electromagnetic tomography (sLORETA; Pascual-Marqui, 2002) was used to estimate the intracerebral electrical sources. As a standard procedure a common average reference transformation (Pascual-Marqui, 2002) is performed before applying the sLORETA algorithm. sLORETA computes electric neuronal activity as current density $\left(\mathrm{A} / \mathrm{m}^{2}\right)$ without assuming a predefined number of active sources. The solution space used in this study and associated leadfield matrix are those implemented in the LORETA-Key software (freely available at http://www.uzh.ch/keyinst/ loreta.htm). This software implements revisited realistic electrode coordinates (Jurcak et al., 2007) and the lead field produced by Fuchs et al. (2002) applying the boundary element method on the MNI-152 (Montreal Neurological Institute). The sLORETA-key anatomical template divides and labels the neocortical (including hippocampus and anterior cingulate cortex) MNI-152 space in 6239 voxels of dimension $5 \mathrm{~mm}^{3}$, based on probabilities returned by the Demon Atlas (Lancaster et al., 2000). The coregistration makes use of the correct translation from the MNI-152 space into the Talairach and Tournoux space.

Region-of-interest analysis. The log-transformed electric current density were calculated for the regions-of-interest (ROIs) for the different frequency bands: delta $(2-3.5 \mathrm{~Hz})$, theta $(4-7.5 \mathrm{~Hz})$, alpha $(8-12 \mathrm{~Hz})$, beta $(13-30 \mathrm{~Hz})$, and gamma $(30.5-44 \mathrm{~Hz})$. The ROIs in the present study are pregenual anterior cingulate cortex, left auditory cortex, and the left parahippocampus. Table 2 provides the MNI coordinate for each ROI. As the voxel-size of each ROI has the dimension $5 \mathrm{~mm}^{3}$, we only used this voxel. For the pregenual anterior cingulate cortex, we do not differentiate between left and right because of their proximity to the midline. Because of volume conduction, laterality is harder to differentiate for areas close to the midline. For the auditory cortex and parahippocampus we only include the left as the whole-brain analysis only showed left-sided activity. The selection of these ROI was based on our hypothesis as introduced in the introduction (a priori) and confirmed by the comparison of activity between the tinnitus groups with hearing loss and tinnitus with a hearing loss (a posteriori).

Model generation. We used the data-mining software Weka (Waikato Environment for Knowledge Analysis v3.7, developed by the University of Waikato Machine Learning Group, http://www.cs.waikato.ac.nz/ml/ weka/; Smith and Frank, 2016) to perform all classification tasks. The Weka software suite contains a library of algorithms that build predictive models by learning from examples provided in user supplied datasets. We use the default settings as the running parameters. Our dataset included for each subject the five frequency bands (i.e., delta, theta, alpha, beta, and gamma) for each ROI (i.e., left auditory cortex, left parahippocampus, and pregenual anterior cingulate cortex). The criterion for correct classification was defined as when subjects were assigned to the correct group based (top-down vs bottom-up) on the model calculated by the WEKA software.

Lagged phase coherence. Coherence and phase synchronization between time series corresponding to different spatial locations are usually interpreted as indicators of the "connectivity". However, any measure of dependence is highly contaminated with an instantaneous, nonphysiological contribution because of volume conduction (Pascual-Marqui, 2007b). However, Pascual-Marqui (2007a) introduced new measures of coherence and phase synchronization taking into accounts only noninstantaneous (lagged) connectivity, effectively removing the confounding factor of volume conduction. Such "lagged phase coherence" between two sources can be interpreted as the amount of crosstalk between the regions contributing to the source activity (Congedo et al., 2010). Since the two components oscillate coherently with a phase lag, the crosstalk can be interpreted as information sharing by axonal transmission. More precisely, the discrete Fourier transform decomposes the signal in a finite series of cosine and sine waves at the Fourier frequencies (Bloomfield, 2000). The lag of the cosine waves with respect to their sine counterparts is inversely proportional to their frequency and amounts to one-quarter of the period; for example, the period of a sinusoidal wave at $10 \mathrm{~Hz}$ is $100 \mathrm{~ms}$. The sine is shifted a quarter of a cycle $(25 \mathrm{~ms})$ with the respect to the cosine. Then the lagged phase coherence at $10 \mathrm{~Hz}$ indicates coherent oscillations with a $25 \mathrm{~ms}$ delay, whereas at $20 \mathrm{~Hz}$ the delay is 12.5 $\mathrm{ms}$, etc. The threshold of significance for a given lagged phase coherence value according to asymptotic results can be found as described by Pascual-Marqui (2007b), where the definition of lagged phase coherence can be found as well. As such, this measure of dependence can be applied to any number of brain areas jointly, i.e., distributed cortical networks, whose activity can be estimated with sLORETA. Measures of linear dependence (coherence) between the multivariate time series are defined. The measures are non-negative, and take the value zero only when there 


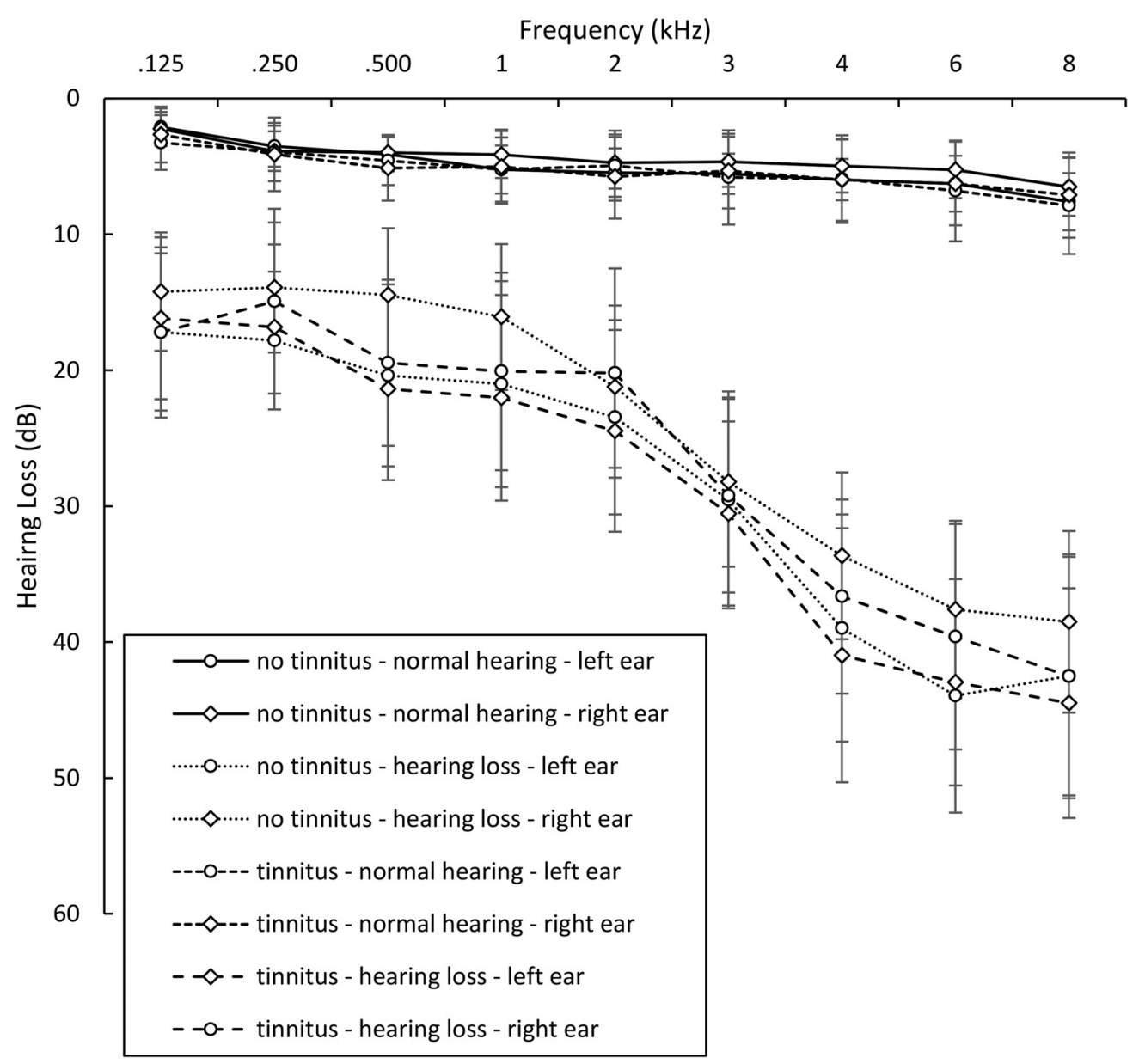

Figure 1. Audiogram for the different groups.

Table 2. ROIs

\begin{tabular}{|c|c|c|c|}
\hline \multirow[b]{2}{*}{ Area } & \multicolumn{3}{|c|}{ ROI, MNI space } \\
\hline & $x$ & $y$ & $z$ \\
\hline pgACC & 0 & 42 & -1 \\
\hline Left AUD & -29 & -31 & -1 \\
\hline Left PHC & -54 & -21 & -9 \\
\hline
\end{tabular}

pgACC, pregenual anterior cingulate cortex; AUD, auditory cortex; PHC, parahippocampus.

is independence and are defined in the frequency domain: delta (2-3.5 $\mathrm{Hz})$, theta $(4-7.5 \mathrm{~Hz})$, alpha $(8-12 \mathrm{~Hz})$, beta $(13-30 \mathrm{~Hz})$, and gamma $(30.5-44 \mathrm{~Hz})$. Based on this principle lagged linear connectivity was calculated. Time-series of current density were extracted for different ROIs using sLORETA. Power in all 6239 voxels was normalized to a power of 1 and $\log$ transformed at each time point. Region of interest values thus reflect the log transformed fraction of total power across all voxels, separately for specific frequencies. ROIs selected were the pregenual anterior cingulate cortex, left auditory cortex, and the left parahippocampus.

Granger causality. Granger causality reflects the strength of effective connectivity (i.e., causal interactions, extracted activity of one area of causal influences of one neural element over another) from one region to another by quantifying how much the signal in the seed region is able to predict the signal in the target region (Granger, 1969; Geweke, 1982). In other words, it can be considered a directional functional connectivity. Granger causality is based on formulating a multivariate autoregressive model and calculating the corresponding partial coherences after setting all irrelevant connections to zero. We decided to use Granger causality as it can be directly applied to any given time series to detect the coupling among empirically sampled neuronal systems (Friston et al., 2013). This can provide insights into the dynamical behavior of a system in spontaneously active "resting" states (Friston et al., 2013). Granger causality is accepted because there is no temporal lag between the responses recorded and their underlying causes and because the data can be sampled at fast timescales. The advantages of Granger causality in furnishing frequency-dependent and multivariate measures have been clearly demonstrated in previous electrophysiology research (Barrett et al., 2012; Bosman et al., 2012). All technical details can be found in (Stokes and Purdon, 2017). In general, the autoregressive coefficients correspond to Granger causality (Granger et al., 1969; Valdes-Sosa et al., 2011). It is defined as the log-ratio between the error variance of a reduced model, which predicts one time series based only on its own past values, and that of the full model, which in addition includes the past values of another time series. It is important to note that Granger causality does not imply anatomical connectivity between regions but directional functional connectivity between two sources. In this study, we look at the effective connectivity between the pregenual anterior cingulate cortex, the left auditory cortex, and the left parahippocampus for the theta frequency band. We selected the theta frequency band based on our previous findings (Vanneste and De Ridder, 2016; a priori) and confirmed by the comparison of functional connectivity outcome (a posteriori).

In addition, we applied generalized partial directed coherence (PDC) and isolated effective coherence (iCoh) to look at the relationship pregenual anterior cingulate cortex, the left auditory cortex, and the left parahippocampus for the theta frequency band to further validate our Granger causality findings. PDC is a measure designed to quantify direct connections that are not confounded by indirect paths, their directionality, and their spectral characteristics (Baccalá and Sameshima, 2001). iCoh consists of estimating the partial coherence under a multivariate 
autoregressive model, followed by setting all irrelevant associations to zero. All technical details we published previously (Pascual-Marqui et al., 2014).

Cross-frequency coupling. Theta-gamma coupling (e.g., by nesting) is proposed to be an effective manner of communication between cortically distant areas (Canolty et al., 2006). To verify whether this theta-gamma nesting is present, it was calculated for the pregenual anterior cingulate cortex, left auditory cortex, and the left parahippocampus cortex using phase-amplitude cross-frequency coupling. Phase-amplitude was chosen over power-power cross-frequency coupling as the former has been shown to reflect a physiological mechanism for effective communication in the human brain (Canolty et al., 2006). Nesting was computed by first obtaining the time-series for the $x, y$, and $z$ components of the sLORETA current for the voxel of each ROI. These are the time-series of the electrical current in the three orthogonal directions in space. Next, these were filtered in the theta $(4-7.5 \mathrm{~Hz})$ and gamma $(30.5-44 \mathrm{~Hz})$ frequency bandpass regions. In each frequency band and for each ROI, a principal component analysis for the overall $x, y, z$ component was computed and the first component was retained for the theta and gamma bands. The Hilbert transform was then computed on the gamma component and the signal envelope retained. Finally, the Pearson correlation between the theta component and the envelope of the gamma envelope was computed for each individual.

\section{Statistical analyses}

Whole brain. The methodology used is a nonparametric permutation test. It is based on estimating, via randomization, the empirical probability distribution for the max-statistic under the null hypothesis comparisons (Nichols and Holmes, 2002). This methodology corrects for multiple testing (i.e., for the collection of tests performed for all voxels, and for all frequency bands). Due to the nonparametric nature of this method, its validity does not rely on any assumption of Gaussianity (Nichols and Holmes, 2002). These whole-brain comparisons were performed by sLORETA through multiple voxel-by-voxel comparisons using a logarithm of $F$ ratio. The significance threshold for all tests was based on a permutation test with 5000 permutations. Comparisons were made between the healthy controls versus tinnitus group with normal hearing, healthy controls versus tinnitus with hearing loss, and between the tinnitus groups with normal hearing loss and tinnitus with hearing loss. To correct for the 4 comparisons $\times$ the 5 frequency bands we used the FDR method (Benjamini and Hochberg, 1995).

ROI. We performed a MANOVA including the log-transformed current density for the pregenual anterior cingulate cortex, left auditory cortex, and left parahippocampus as dependent variables and hearing (normal hearing vs hearing loss) and phantom percept (tinnitus vs no tinnitus) as independent variables for the alpha frequency as well for the gamma frequency band. To correct for the two MANOVA test we applied a Holm-Bonferroni method (Holm, 1979). If the outcome of the MANOVA was significant, a univariate ANOVA was conducted for the different regions separately. A correction for multiple correction using the Holm-Bonferroni method was applied to correct for the different univariate ANOVAs (Holm, 1979). In addition, simple contrast analyses were conducted to look at specific effects using a Bonferroni correction for multiple comparisons.

In addition, Pearson correlations were calculated between the ROI and the subjective loudness for the gamma frequency band for tinnitus patients that have normal hearing as well as hearing loss. This analysis was corrected for pairwise comparisons using a Bonferroni correction ( $p=$ $0.025)$.

For tinnitus patients with normal hearing, we hypothesize that the noise-cancelling mechanism is deficient, which will slow down the alpha to theta activity in the pregenual anterior cingulate cortex. To look at this relationship, we calculated the theta/alpha ratio for the pregenual anterior cingulate cortex. This theta-alpha ratio was correlated with the subjective loudness using a Pearson correlation. To correct for pairwise comparisons, we used a Bonferroni correction $(p=0.025)$.

Model generation. We used a linear logistic regression-based classifier as the classification method. A tenfold cross-validation was performed on the full dataset (Vanneste et al., 2018b). The measurements of model accuracy calculated by the $k$-fold cross-validation technique include the true-positive ratio, false-negative ratio root mean squared error (RMSE), mean average error (MAE), and $\kappa$-statistic. The true-positive ratio was calculated as the ratio of the total number of correctly classified positive instances (in this case, positive refers to tinnitus patients) over the total number of positive instances in the testing sample. The RMSE is a measure of how well the machine learns the model, and it was calculated by taking the square root of the average of the residuals (errors not explained by the regression equation) over the total sample size. The MAE is simply the average of residuals over the total sample size. The $\kappa$-statistic compares the model's observed accuracy with its expected (chance) accuracy by taking the difference in observed and expected accuracy over $1-$ expected accuracy.

Lagged phase coherence. Lagged phase coherence or functional connectivity contrast were calculated for the different frequency bands (delta, theta, alpha, beta, and gamma) for the four groups (normal hearing/ no tinnitus, hearing loss/no tinnitus, normal hearing/tinnitus, and hearing loss/tinnitus). The significance threshold was based on a permutation test with 5000 permutations. This methodology corrects for multiple testing (i.e., for the collection of tests performed for all voxels, and for all frequency bands). Comparisons were made between the healthy controls versus tinnitus group with normal hearing, healthy controls versus tinnitus with hearing loss, and between the tinnitus groups with normal hearing loss and tinnitus with hearing loss. To correct for the 4 comparisons $\times$ the 5 frequency bands we are using the FDR method (Benjamini and Hochberg, 1995).

Granger causality. We performed a MANOVA including the Granger causality for the effective connectivity [pregenual anterior cingulate cortex $\rightarrow$ left auditory cortex (pgACC $\rightarrow$ AUD), pregenual anterior cingulate cortex $\rightarrow$ left parahippocampus (pgACC $\rightarrow$ PHC), left auditory cortex $\rightarrow$ pregenual anterior cingulate cortex (AUD $\rightarrow$ pgACC), pregenual anterior cingulate cortex $\rightarrow$ pregenual anterior cingulate cortex ( $\mathrm{PHC} \rightarrow$ pgACC)] as dependent variables, and hearing (normal hearing vs hearing loss) and phantom percept (tinnitus vs no tinnitus) as independent variables for the theta frequency band. If the outcome of the MANOVA was significant, a univariate ANOVA was conducted for the different connections separately. A correction for multiple corrections was applied for the different univariate ANOVAs using the Holm-Bonferroni correction (Holm, 1979). In addition, simple contrast analyses were conducted to look at specific effects using a Bonferroni correction for multiple comparison. A similar analysis was applied for PDC and iCoh. To correct for the three MANOVAs (granger, PDC, and iCoh) conducted we applied a Holm-Bonferroni method (Holm, 1979).

Phase-amplitude coupling. We performed a MANOVA including the phase-amplitude coupling for theta-gamma for the pregenual anterior cingulate cortex, auditory cortex and parahippocampus as dependent variables and hearing (normal hearing vs hearing loss) and phantom percept (tinnitus vs no tinnitus) as independent variables. If the outcome of the MANOVA was significant, a univariate ANOVA was conducted for the different cross-frequency coupling separately using the Holm-Bonferroni correction for multiple comparison (Holm, 1979). If the outcome of the univariate ANOVA was significant, a simple contrast analysis was conducted to look if there was a difference between normal hearing versus hearing loss for participants with no tinnitus and with tinnitus, respectively. A Bonferroni correction for multiple comparisons was conducted to correct for the pairwise comparisons $(p=0.025)$.

\section{Results}

\section{Whole-brain analysis}

No tinnitus: hearing loss versus normal hearing

Participants with hearing loss but no tinnitus demonstrate significantly increased activity in the pregenual anterior cingulate cortex and the adjacent medial superior frontal gyrus $(F=2.58, p=$ 0.011 ) for the alpha frequency band compared with participants with normal hearing but no tinnitus (Fig. $2 a$ ). No significant effects were observed for the delta, theta, beta, and gamma frequency bands. 
a
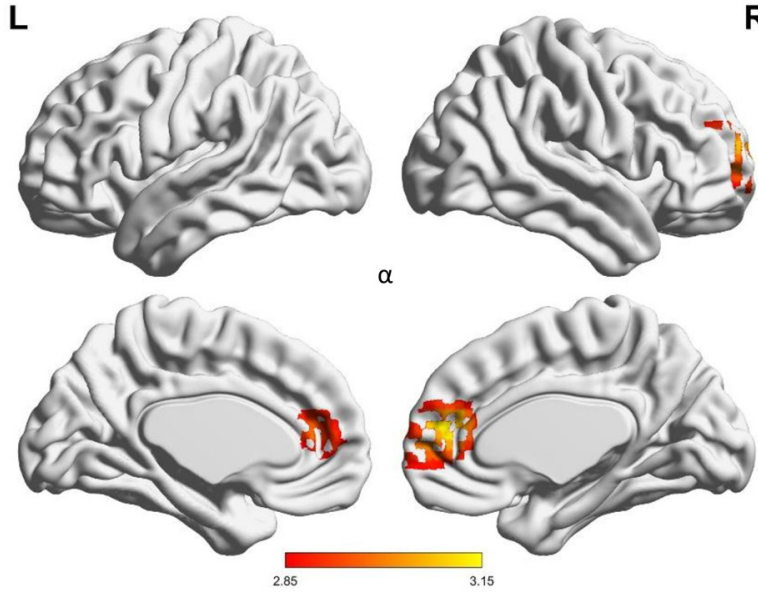

c
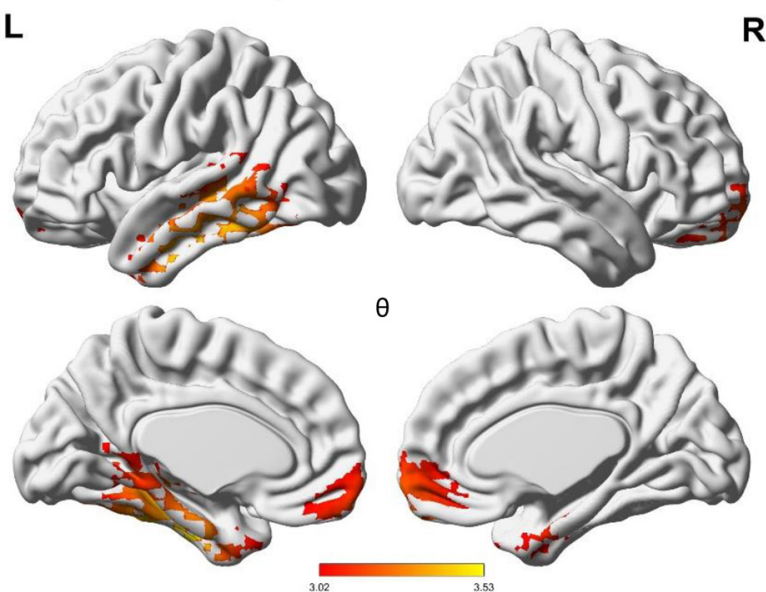

$\mathbf{R}$ b

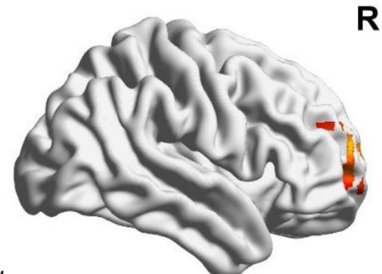

$\mathbf{R}$

$\mathbf{L}$

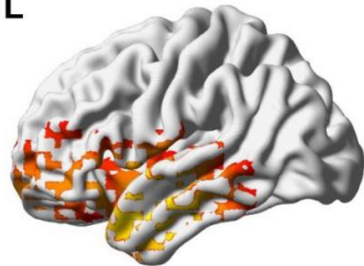

Normal hearing: tinnitus vs no tinnitus
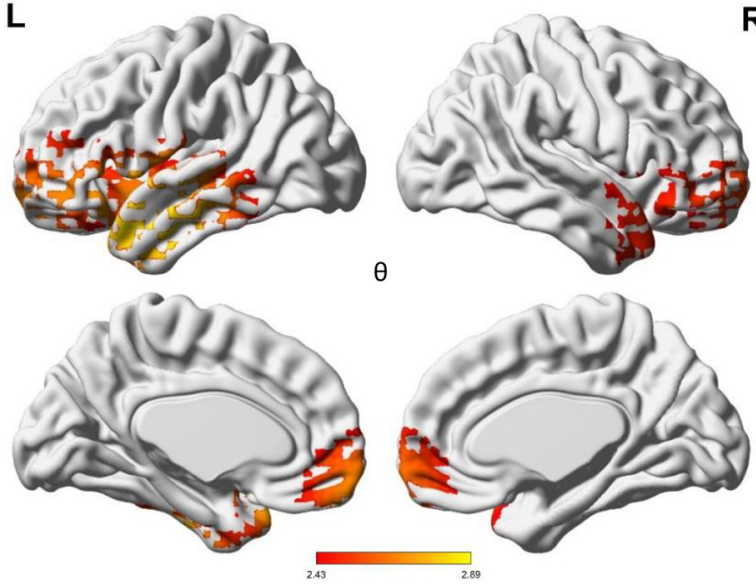

$\mathbf{R}$

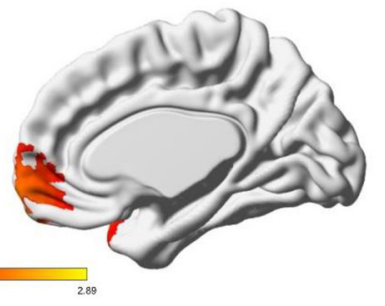

d

$\mathbf{L}$

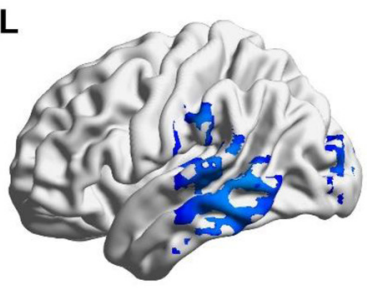

Tinnitus: hearing loss vs. normal hearing

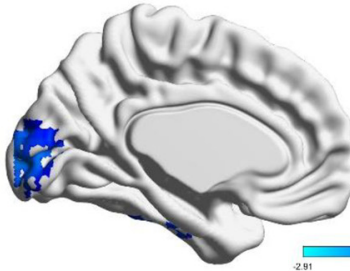

Y

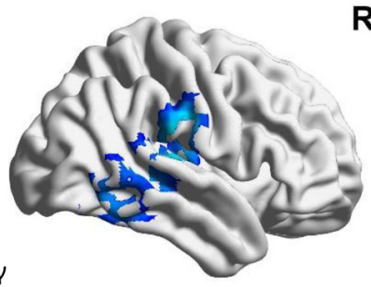

$\mathbf{R}$

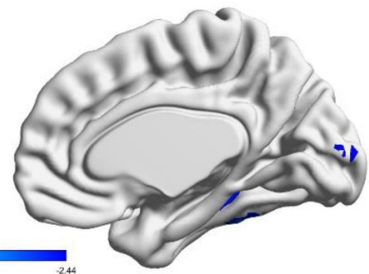

Figure 2. Whole-brain analysis. $\boldsymbol{a}$, For participants with no tinnitus, a comparison between hearing loss vs normal hearing revealed increased significant synchronized activity in the pregenual anterior cingulate cortex for the alpha frequency band for participants with hearing loss. $\boldsymbol{b}$, For participants with mild to normal hearing, a comparison between tinnitus versus no tinnitus participants demonstrated a significant effect for the theta frequency bands over the pregenual anterior cingulate cortex and left auditory cortex for the theta frequency for participants with tinnitus. c, For participants with hearing loss, a comparison between tinnitus versus no tinnitus participants revealed increased synchronized activity in the left parahippocampus extending in to the inferior and middle temporal cortex as well as pregenual anterior cingulate cortex for the theta frequency band for participants with tinnitus. $d$, For participants with tinnitus, a comparison between hearing loss and normal hearing participants showed significant decrease synchronized activity was obtained in the gamma frequency range at the left and right auditory cortex for the participants with hearing loss.

\section{Normal hearing: tinnitus versus no tinnitus}

A comparison between normal hearing subjects with and without tinnitus revealed a significant effect for the theta frequency bands $(F=2.43, p=0.015)$, demonstrating that tinnitus subjects have increased activity over the pregenual anterior cingulate cortex, frontopolar cortex, and left auditory cortex for the theta frequency band (Fig. 2b). No significant effects were observed for the delta, alpha, beta, and gamma frequency bands.

\section{Hearing loss: tinnitus versus no tinnitus}

For participants with hearing loss, a comparison between tinnitus and no tinnitus subjects revealed increased activity for tinnitus subjects in the left parahippocampus, the inferior and middle temporal cortex, pregenual anterior cingulate cortex, and frontopolar cortex $(F=3.02, p=0.001)$ for the theta frequency band (Fig. 2c). No significant effects were observed for the delta, alpha, beta, and gamma frequency bands.

Tinnitus: hearing loss versus normal hearing

A comparison between tinnitus participants with hearing loss compared with normal hearing who have tinnitus demonstrates significantly decreased activity in the gamma frequency range at the left and right auditory cortex, supramarginal gyrus, posterior middle and inferior temporal gyri $(F=-2.44, p=0.018)$, as well as cuneus for the participants with hearing loss (Fig. $2 d$ ). No significant effects were observed for the delta, theta, alpha, and beta frequency bands. These results were not affected after controlling for tinnitus lateralization.

\section{ROI analysis}

To better understand the interaction between hearing (normal hearing vs hearing loss) and phantom perception (tinnitus vs no tinnitus), we conducted a ROI analysis for the alpha and gamma frequency band including the pregenual anterior cingulate cortex, left auditory cortex, and left parahippocampus based on our hypothesis and confirmed by the whole-brain analysis.

A MANOVA including the log-transformed current density for the pregenual anterior cingulate cortex, left auditory cortex, and left parahippocampus as dependent variables and hearing (normal hearing vs hearing loss) and phantom percept (tinnitus vs no tinnitus) as independent variables for the alpha frequency 
band showed an overall interaction effect $(F=2.65, p=0.031$, $\eta^{2}=0.11$ ). A univariate ANOVA shows that an effect was only obtained for the pregenual anterior cingulate cortex $(F=4.15$, $p=0.045, \eta^{2}=0.07$ ), showing a significant increase in current density at the alpha frequency band for subjects with hearing loss who have no tinnitus compared with subjects with normal hearing and subjects with tinnitus with and without hearing loss $(p<$ 0.05 ). This effect does however not survival correction for multiple comparisons. A univariate ANOVA did not show a significant effect at the auditory cortex $(F=1.21, p=0.28)$ and parahippocampus $(F=0.16, p=0.69)$ for the alpha frequency band. For a summary, see Figure 3.

A similar analysis was applied to the gamma frequency band, which revealed an overall interaction effect between hearing (normal hearing vs hearing loss) and phantom percept (tinnitus vs no tinnitus; $F=3.15, p=0.013, \eta^{2}=0.13$ ). A univariate ANOVA did not show a significant interaction effect at the pregenual anterior cingulate cortex $(F=1.95, p=0.34)$ for the gamma frequency band. An univariate ANOVA shows an interaction effect for the auditory cortex $(F=8.72, p=0.004)$, indicating increased current density for participants with tinnitus and normal hearing compared with tinnitus participants with hearing loss $(p<0.001)$ as well as participants with no tinnitus with normal hearing $(p<0.001)$ or hearing loss using a simple contrast analysis $(p<0.001)$. Furthermore, a significant interaction effect was obtained for the parahippocampus $(F=6.59$, $\left.p=0.013, \eta^{2}=0.09\right)$ at the gamma frequency band showing increased current density for participants with tinnitus and hearing loss compared with tinnitus participants with normal hearing ( $p=0.011$ ), as well as participants with no tinnitus independent of the hearing loss (normal hearing vs hearing loss; $p<0.001$ ). To verify whether lateralization (unilateral vs bilateral tinnitus) has an effect on the outcome of the parahippocampus, we conducted an additional ANOVA with hearing (normal hearing vs hearing loss) and tinnitus lateralization (unilateral vs bilateral) as independent variables and left parahippocampus as the dependent variable. This analysis confirmed an effect for hearing loss $\left(F=5.12, p=0.031, \eta^{2}=\right.$ $0.16)$ demonstrating increased current density for tinnitus participants with hearing loss $(M=-4.42, \mathrm{SD}=0.61)$ compared with tinnitus participants with normal hearing $(M=$ $-5.02, \mathrm{SD}=0.91)$. No effect was obtained for tinnitus lateralization $(F=0.01, p=0.91)$ or for the interaction effect between hearing and tinnitus lateralization $(F=0.12, p=$ 0.74). For a summary, see Figure 3.

For the tinnitus participants, a Pearson correlation analysis further showed a positive, marginally significant correlation between the subjective loudness of the tinnitus and logtransformed current density at the pregenual anterior cingulate cortex $(r=0.44, p=0.067)$ for the gamma frequency band for participants with normal hearing loss. This effect remains after correcting for distress $(r=0.44, p=0.08)$. However, after a Bonferroni correction for multiple comparisons, the effect was lost. A similar correlation between the subjective loudness of the tinnitus and the log-transformed current density at the pregenual anterior cingulate cortex for participants with hearing loss did not show an effect. For auditory cortex $(r=0.55, p=0.008)$, a positive correlation was obtained between the subjective loudness of the tinnitus and the log-transformed current density at the gamma frequency band for participants with normal hearing. This effect remains after correcting for distress $(r=0.57, p=$ 0.018). However, after a Bonferroni correction for multiple comparison, the effect was lost. For tinnitus participants with hearing loss, no significant correlation $(r=-0.03, p=0.91)$ was obtained between the subjective loudness of the tinnitus and log-transformed current density at the auditory cortex. For the parahippocampus, a positive correlation between the subjective loudness and logtransformed current density $(r=0.76, p<0.001)$ for the gamma frequency band for subjects with hearing loss, which remains after Bonferroni correction. This effect remains after correcting for distress $(r=0.78, p<0.001)$. No effect was obtained between the subjective and log-transformed current density at the parahippocampus ( $r=0.13, p=0.61$ ) for tinnitus participants with normal hearing. For a summary, see Figure 3.

To further verify whether the effect obtained could be explained by tinnitus related distress, we applied a Pearson correlation analysis with the log-transformed current density at the pregenual anterior cingulate cortex, the left auditory cortex and the left parahippocampus for the gamma frequency band for participants with normal hearing loss and for participants with hearing loss, separately. These analyses did not show a significant effect $(r=-0.33$ to $0.09, p=0.18-0.73)$.

A Pearson correlation between the theta/alpha ratio for the pregenual anterior cingulate cortex and subjective loudness for tinnitus patients with normal hearing showed a significant positive correlation $(r=0.61, p=0.007)$. This effect remained after correction for multiple comparisons. This suggests that decreased alpha activity and increased theta activity corresponds with increased subjective loudness. No effect was obtained between the theta/alpha ratio and subjective loudness for tinnitus patients with hearing loss $(r=0.31, p=0.21$; Fig. 4$)$.

\section{Model generation}

To verify whether the activity in the pregenual anterior cingulate cortex, the auditory cortex, and the parahippocampus can classify tinnitus in terms of bottom-up versus top-down, we applied simple logistic regression to classify the data into two classes using simple logistic regression. Using simple logistic regression shows a significant effect $\left(\chi_{(3)}^{2}=24.72, p<0.001\right.$, Nagelkerke $R^{2}=$ $0.66)$ indicating we can differentiate between bottom-up and top-down tinnitus. The true-positive rate was $80.55 \%$ and the false-negative rate was $19.45 \%$. The ROC was $84.6 \%$, the $\kappa$-statistic 0.61, MAE 0.31, and RMSE 0.39 (see Table 3).

To confirm these results, we applied a discriminant analysis (Wilk's $\lambda_{(3)}=0.51, \chi_{(3)}^{2}=22.06, p<0.001$ ) explaining $70.2 \%$ of variance based on the canonical correlation. The ANOVA shows that the pregenual anterior cingulate cortex for the theta frequency band (Wilk's $\lambda_{(1,34)}=0.71, F_{(1,34)}=14.11, p=0.001$ ), the left auditory cortex for the gamma frequency band (Wilk's $\left.\lambda_{(1,34)}=0.79, F_{(1,34)}=8.97, p=0.005\right)$, and the left parahippocampus for the gamma frequency band (Wilk's $\lambda_{(1,34)}=0.88$, $\left.F_{(1,34)}=4.61, p=0.039\right)$.

\section{Functional connectivity: lagged phase coherence}

To understand the relationship the pregenual anterior cingulate cortex, left auditory cortex, and left parahippocampus, we compare participants with and without tinnitus that have or do not have hearing loss.

\section{No tinnitus: hearing loss versus normal hearing}

For participants with no tinnitus, a comparison between normal hearing and hearing loss subjects revealed a significant effect in phase coherence for the alpha frequency band $(F=3.61, p<0.001)$. That is, participants with hearing loss have increased coherence between the pregenual anterior cingulate cortex and the left auditory cortex compared with participants with no tinnitus but normal hearing 

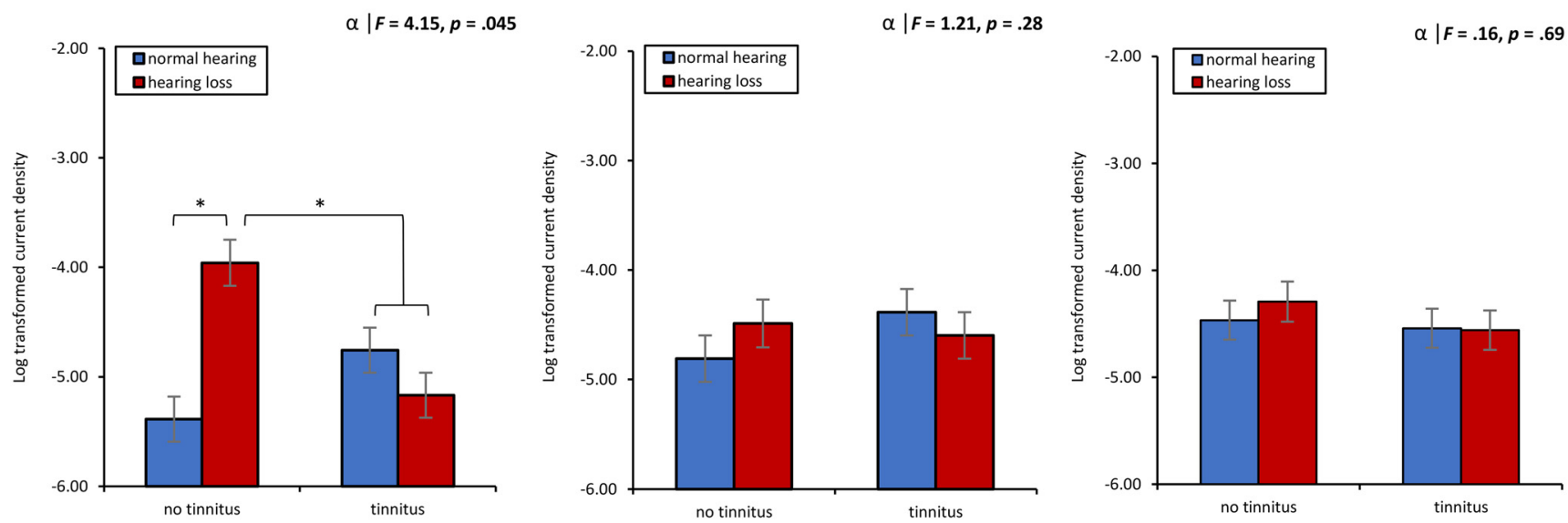

Overall effect $F=3.15, p=.013$
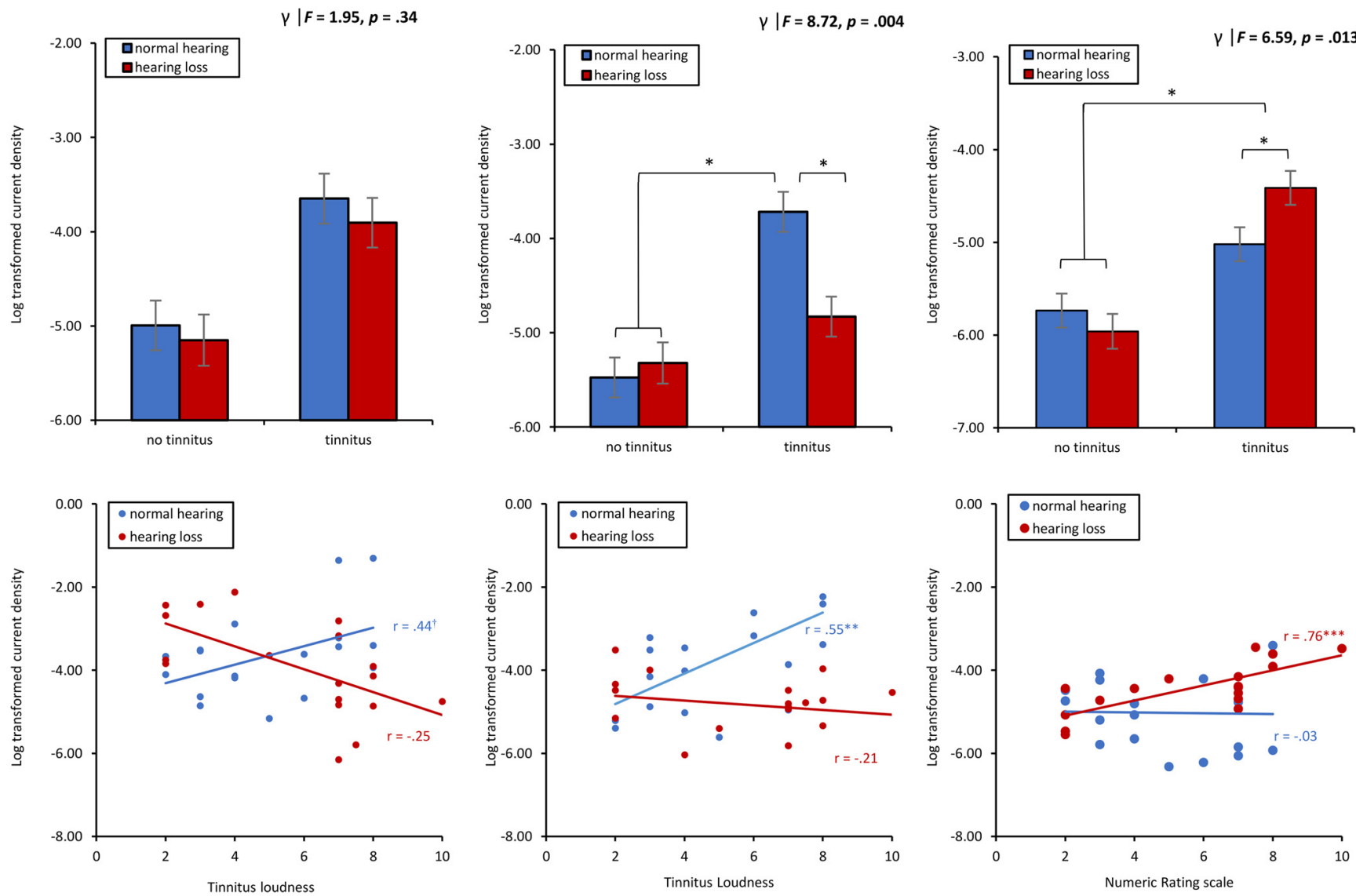

Figure 3. ROl analysis. Top, The interaction effect of hearing (hearing loss vs normal hearing) and phantom percept (tinnitus vs no tinnitus) on the log-transformed current density for the pregenual anterior cingulate cortex, left auditory cortex, and left parahippocampus for the alpha frequency band. Middle, The interaction effect of hearing (hearing loss vs normal hearing) and phantom percept (tinnitus vs no tinnitus) on the log-transformed current density for the pregenual anterior cingulate cortex, left auditory cortex, and left parahippocampus for the gamma frequency band. Bottom, The Pearson correlation analysis between the tinnitus loudness as measured with the numeric rating scale and log-transformed current density for the pregenual anterior cingulate cortex, left auditory cortex, and left parahippocampus for the gamma frequency band for tinnitus patient with normal hearing loss as well as hearing loss. ${ }^{*} p<.05,{ }^{* *} p<.01,{ }^{* * *} p<.001$.

(Fig. 5a). No significant effects were observed for the delta, theta, beta, and gamma frequency bands.

Normal hearing: tinnitus versus no tinnitus

A comparison between normal hearing subjects with and without tinnitus revealed a significant difference in phase coherence for the theta frequency band. Specifically, it revealed decreased coherence between the left parahippocampus and left auditory cor- tex, as well as between the left auditory cortex and the pregenual anterior cingulate cortex for tinnitus subjects $(F=2.97, p=$ 0.012; Fig. $5 b$ ). No significant effect was observed for the delta, alpha, beta, and gamma frequency bands.

Hearing loss: tinnitus versus no tinnitus

For hearing loss, a comparison between participants with and without tinnitus revealed a significant difference in phase coher- 
ence for the theta frequency band ( $F=$ 3.03, $p=0.010)$. This comparison showed that participants with tinnitus have a decrease in coherence between the pregenual anterior cingulate cortex and the left auditory cortex and left parahippocampus, respectively. Furthermore, there was increased connectivity between the left auditory cortex and left parahippocampus for tinnitus participants (Fig. $5 c$ ). No significant effect was observed for the delta, alpha, beta, and gamma frequency bands.

Tinnitus: hearing loss versus normal hearing

For tinnitus, a comparison between participants with hearing loss and normal hearing revealed a significant difference in phase coherence for the theta frequency band $(F=3.24, p=0.007)$. For this frequency band, we found that participants with mild hearing loss have a decrease in coherence between the pregenual anterior cingulate cortex and the left auditory cortex and between the pregenual anterior cingulate cortex and the left parahippocampus (Fig. 5d). Furthermore, there was an increase in connectivity between the left auditory cortex and left parahippocampus for participants with mild hearing loss. No significant effect was observed for the delta, alpha, beta, and gamma frequency bands.

Effective connectivity: granger causality, PDC, iCoh

Based on functional connectivity, we look specifically at the directionality between the pregenual anterior cingulate cortex, the left auditory cortex, and the left parahippocampus for participants with and without tinnitus that have or do not have hearing loss for the theta frequency band.

A MANOVA including the Granger causality for the effective connectivity (pgACC $\rightarrow$ AUD, pgACC $\rightarrow$ PHC, AUD $\rightarrow$ pgACC, $\mathrm{PHC} \rightarrow \mathrm{pgACC}$ ) as dependent variables and hearing (normal hearing vs hearing loss) and phantom perception (tinnitus vs no tinnitus) as independent variables for the theta frequency band showed an overall interaction $\left(F=5.86, p<0.001, \eta^{2}=0.21\right.$; Fig. 6). A univariate ANOVA shows a significant interaction effect for $\operatorname{pgACC} \rightarrow \operatorname{AUD}\left(F=3.83, p=0.048, \eta^{2}=0.05\right)$, revealing that tinnitus patients with normal hearing have a decrease in connectivity strength from the pgACC to the auditory cortex compared with tinnitus patients with hearing loss $(p<$ $0.05)$. For participants who have no tinnitus, no difference was observed when they have normal hearing compared with hearing loss. A univariate ANOVA for pgACC $\rightarrow$ PHC shows a significant interaction effect $\left(F=4.28, p=0.039, \eta^{2}=0.06\right)$, revealing that tinnitus patients with hearing loss have a decrease in connectivity strength compared with tinnitus patients with normal hearing $(p<0.05)$. For participants who have no tinnitus, no difference was observed when they have normal hearing compared with hearing loss. For $\mathrm{PHC} \rightarrow$ pgACC, we found a significant interaction effect $(F=$ $4.16, p=0.041, \eta^{2}=0.06$ ), revealing increased effective connectivity for participants with no tinnitus and normal hearing
Table 3. Classifier

\begin{tabular}{llll}
\hline & $\beta$ & Wald & $P$ \\
\hline Constant & 2.12 & 0.16 & 0.690 \\
$\theta-$ pgACC & 0.25 & 4.24 & 0.039 \\
$\gamma$-AUD & 6.61 & 3.81 & 0.045 \\
$\gamma$-PHC & 6.24 & 3.94 & 0.043 \\
\hline
\end{tabular}

pgACC, pregenual anterior cingulate cortex; AUD, auditory cortex; PHC, parahippocampus.

compared with participants with no tinnitus and hearing loss $(p<0.05)$. No effect was obtained for PHC $\rightarrow$ pgACC when comparing participants with tinnitus and normal hearing compared with participants with tinnitus and hearing loss. No significant effect was obtained for AUD $\rightarrow \operatorname{pgACC}(F=0.10, p=0.76)$. These findings do not remain after applying a Holm-Bonferroni correction.

A similar MANOVA for PDC including the effective connectivity (pgACC $\rightarrow$ AUD, pgACC $\rightarrow$ PHC, AUD $\rightarrow$ pgACC, $\mathrm{PHC} \rightarrow \mathrm{pgACC}$ ) as dependent variables and hearing (normal hearing vs hearing loss) and phantom percept (tinnitus vs no tinnitus) as independent variables for the theta frequency band again showed an overall interaction $\left(F=4.86, p=0.004, \eta^{2}=\right.$ 0.18 ; Fig. 6). A univariate ANOVA shows a significant interaction effect for $\operatorname{pgACC} \rightarrow \operatorname{AUD}\left(F=4.76, p=0.033, \eta^{2}=0.07\right)$, indicating that tinnitus patients with normal hearing have a decrease in connectivity strength from the pgACC to the auditory cortex compared with tinnitus patients with hearing loss $(p<0.05)$. For participants who have no tinnitus, no difference was observed between the normal hearing and the hearing loss group. A univariate ANOVA for pgACC $\rightarrow$ PHC shows a significant interaction effect $\left(F=5.68, p=0.020, \eta^{2}=0.08\right)$, showing that tinnitus patients with hearing loss have a decrease in connectivity strength compared with tinnitus patients with normal hearing $(p<0.05)$. For participants who have no tinnitus, no difference was ob- 


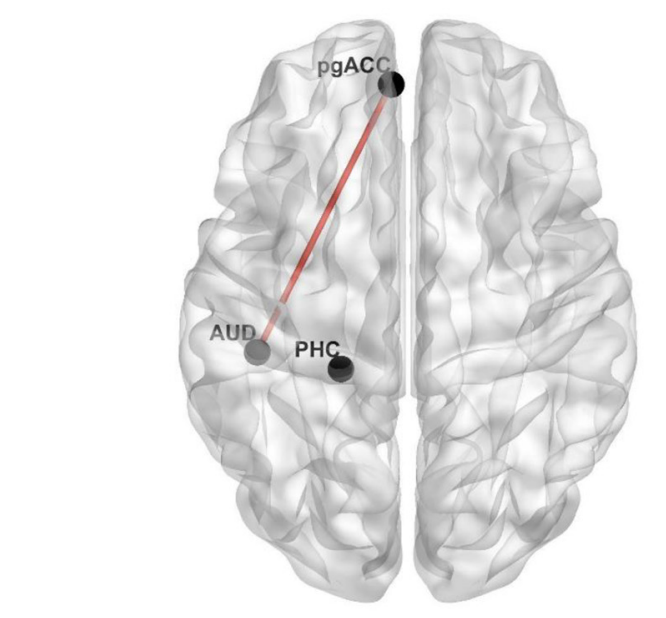

\section{C} Hearing loss: tinnitus vs no tinnitus

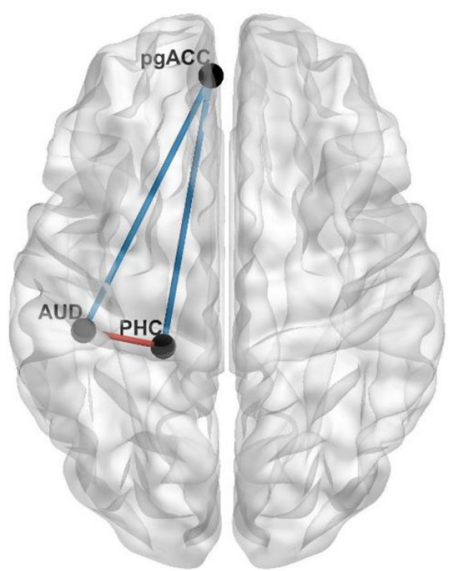

$\alpha$

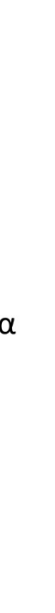

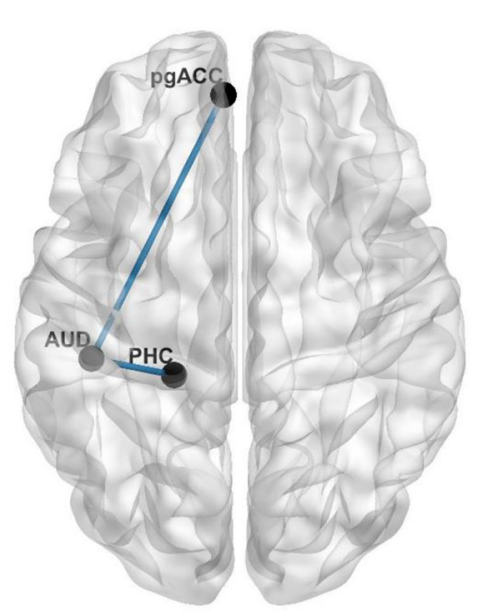

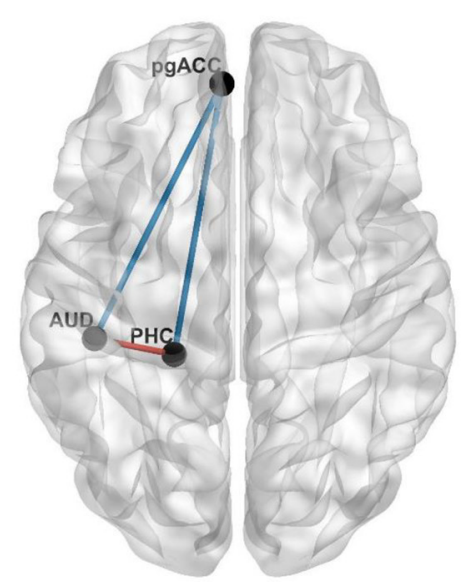

Figure 5. Functional connectivity. $\boldsymbol{a}$, For participants with no tinnitus, a comparison between hearing loss versus normal hearing revealed increased coherence between the pregenual anterior cingulate cortex and the left auditory cortex for the participants with hearing loss for the alpha frequency band. $\boldsymbol{b}$, For participants with normal hearing, a comparison between tinnitus versus no tinnitus participants demonstrated a significant effect for the theta frequency band, revealing decrease coherence between the pregenual anterior cingulate cortex and the left auditory cortex and between the left auditory cortex and left parahippocampus, for tinnitus participants. c, For participants with hearing loss a comparison between tinnitus versus no tinnitus participants revealed a significant difference in phase coherence for the theta frequency band demonstrating that participants with tinnitus have decrease in coherence between the pregenual anterior cingulate cortex and the left auditory cortex and left parahippocampus, respectively as well as increased connectivity between the left auditory cortex and left parahippocampus for tinnitus participants. $\boldsymbol{d}$, For participants with tinnitus, a comparison between hearing loss and normal hearing participants showed decrease in coherence between the pregenual anterior cingulate cortex and the left auditory cortex and left parahippocampus, respectively, and increased connectivity between the left auditory cortex and left parahippocampus for participant with hearing loss in the theta frequency band.

served between normal hearing and hearing loss. For PHC $\rightarrow$ pgACC, we found a significant interaction effect $(F=$ $\left.4.37, p=0.040, \eta^{2}=0.06\right)$, revealing increased effective connectivity for participants with no tinnitus and normal hearing compared with participants with no tinnitus and hearing loss $(p<$ 0.05 ). No effect was obtained for PHC $\rightarrow$ pgACC when comparing participants with tinnitus and normal hearing compared with participants with tinnitus and hearing loss. No significant effect was obtained for AUD $\rightarrow \operatorname{pgACC}(F=0.37, p=0.55)$. These findings do not remain after applying a Holm-Bonferroni correction.

For iCoh, again MANOVA including the effective connectivity (pgACC $\rightarrow$ AUD, $\quad$ pgACC $\rightarrow$ PHC, AUD $\rightarrow$ pgACC, $\mathrm{PHC} \rightarrow$ pgACC) as dependent variables and hearing (normal hearing vs hearing loss) and phantom percept (tinnitus vs no tinnitus) as independent variables for the theta frequency band showed an overall interaction $\left(F=6.87, p<0.001, \eta^{2}=0.24\right.$; Fig. 6). A univariate ANOVA shows a significant interaction ef- fect for pgACC $\rightarrow$ AUD $\left(F=9.49, p=0.003, \eta^{2}=0.12\right)$, revealing that compared with tinnitus patients with hearing loss tinnitus, tinnitus patients with normal hearing have a decrease in connectivity strength from the pgACC $(p<0.05)$. For participants who have no tinnitus. A univariate ANOVA for pgAC$\mathrm{C} \rightarrow \mathrm{PHC}$ shows a significant interaction effect $(F=5.57, p=$ $0.021, \eta^{2}=0.08$ ), showing that tinnitus patients with hearing loss have a decrease in connectivity strength compared with tinnitus patients with normal hearing $(p<0.05)$. For participants who have no tinnitus, no difference was observed when they have normal hearing compared with hearing loss. For PHC $\rightarrow$ pgACC, we found a significant interaction effect $(F=15.18, p<0.001$, $\left.\eta^{2}=0.18\right)$, revealing increased effective connectivity for participants with no tinnitus and normal hearing compared with participants with no tinnitus and hearing loss $(p<0.05)$. No effect was obtained for $\mathrm{PHC} \rightarrow$ pgACC when comparing participants with tinnitus and normal hearing compared with participants with tinnitus and hearing loss. No significant effect was obtained 


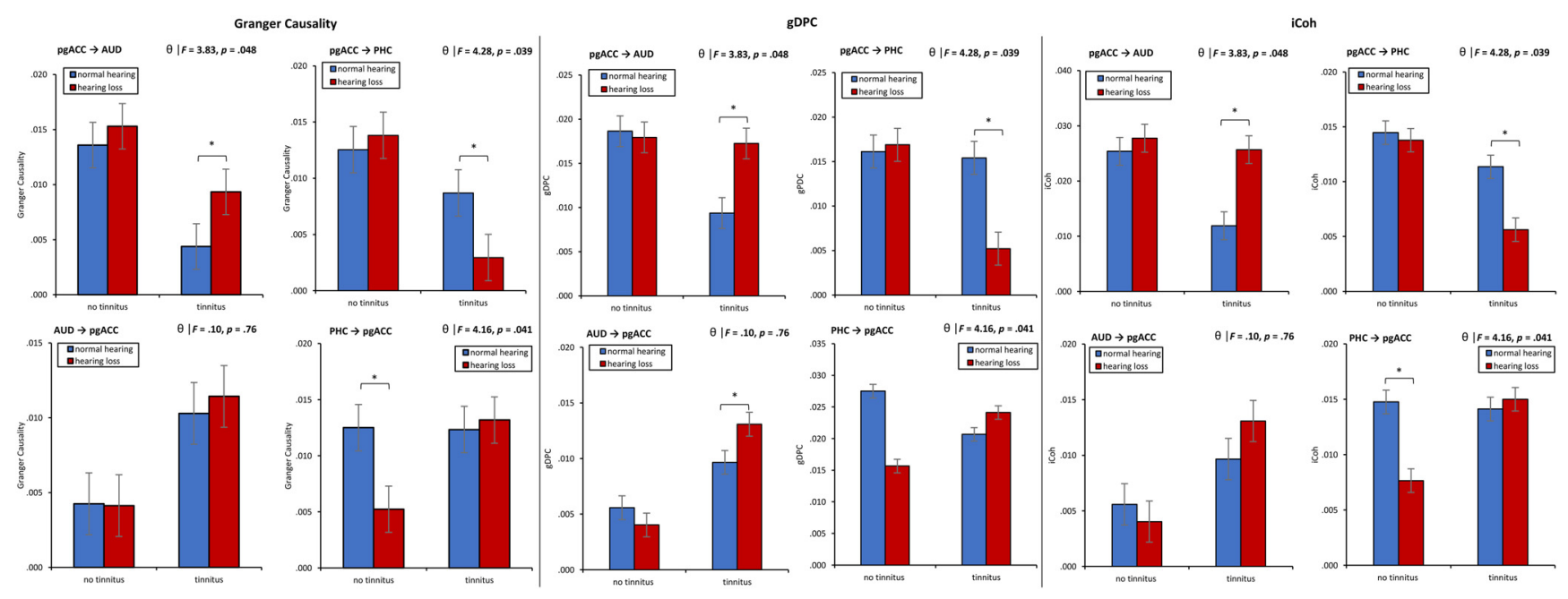

Figure 6. Effective connectivity. Granger causality: Top, Left, The interaction for pgACC $\rightarrow$ AUD. Top, Right, The interaction for pgACC $\rightarrow P H C$. Bottom, Left, The interaction for AUD $\rightarrow$ pgACC. Bottom, Right, The interaction for PHC $\rightarrow$ pgACC. Top, Left, The interaction for pgACC $\rightarrow$ AUD. gDPC: Top, Left, the interaction for pgACC $\rightarrow$ AUD. Top, Right, The interaction for pgACC $\rightarrow$ PHC. Bottom, Left, The interaction for AUD $\rightarrow$ pgACC. Bottom, Right, The interaction for PHC $\rightarrow$ pgACC. Top, Left, The interaction for pgACC $\rightarrow$ AUD. iCoh: Top, Left, The interaction for pgACC $\rightarrow$ AUD. Top, Right, The interaction for pgACC $\rightarrow$ PHC. Bottom, Left, The interaction for AUD $\rightarrow$ pgACC. Bottom, Right, The interaction for PHC $\rightarrow$ pgACC. Top, Left, The interaction for $p g A C C \rightarrow A U D$. ${ }^{*} p<.05$.
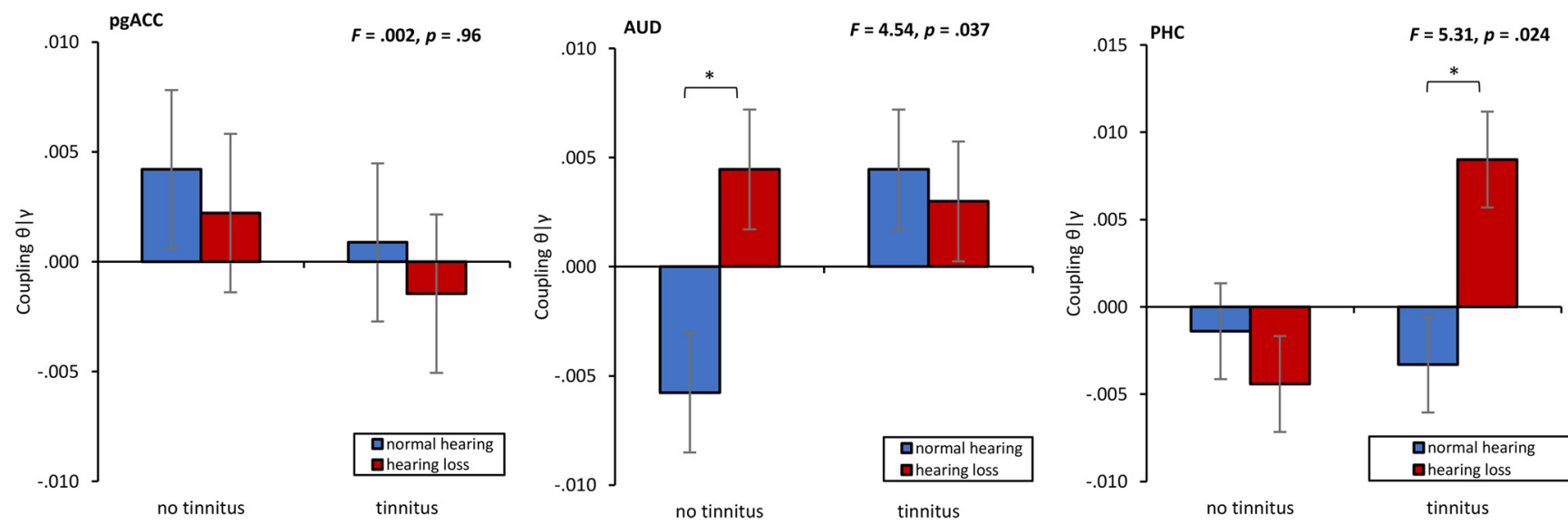

Figure 7. Phase-amplitude coupling for theta- gamma for the pregenual anterior cingulate cortex, left auditory cortex, and left parahippocampus moderated by hearing (hearing loss vs normal hearing) and phantom percept (tinnitus vs no tinnitus). ${ }^{*} p<.05$.

for $\mathrm{AUD} \rightarrow \operatorname{pgACC}(F=1.80, p=0.18)$. These findings do not remain after applying a Holm-Bonferroni correction.

\section{Phase-amplitude coupling}

To better understand how this communication between the pregenual anterior cingulate cortex, left auditory cortex, and left parahippocampus for participants with and without tinnitus that have or do not have hearing loss we look at the theta-gamma coupling. Theta-gamma is proposed to be an effective manner of communication between cortically distant areas (Canolty et al., 2006).

A MANOVA including the phase-amplitude coupling for theta-gamma for the pregenual anterior cingulate cortex, auditory cortex, and parahippocampus as dependent variables and hearing (normal hearing vs hearing loss) and phantom percept (tinnitus vs no tinnitus) as independent variables shows an overall interaction $\left(F=2.78, p=0.048, \eta^{2}=0.11\right.$; Fig. 7$)$. A univariate ANOVA shows a significant effect for the auditory cortex $\left(F=4.54, p=0.037, \eta^{2}=0.06\right)$, showing a significant increase in coupling for subjects with hearing loss and no tinnitus compared with subjects with normal hearing $(p=0.01)$. No significant difference in coupling was demonstrated between subjects with tinnitus depending hearing loss or no hearing loss. For the PHC, a univariate ANOVA yielded a significant interaction effect $(F=$ 5.31, $\left.p=0.024, \eta^{2}=0.07\right)$, showing a significant increase in coupling for subjects with hearing loss and tinnitus compared with subjects with normal hearing $(p=0.012)$. No significant difference in coupling was demonstrated between subjects with no tinnitus based on whether they have hearing loss. A univariate ANOVA did no show a significant effect for the pregenual anterior cingulate cortex. Although the effects shown confirm previous findings (Vanneste and De Ridder, 2016; Vanneste et al., 2018a,b), these findings do not remain after applying a HolmBonferroni correction.

\section{Discussion}

In the present study, we investigated whether tinnitus can be associated with bottom-up deafferentation and/or a deficient top-down noise-cancelling mechanism using resting state EEG. Resting state EEG is an important technique that provides direct information regarding underlying neuronal activity. The advantage of EEG is that it is collected in quiet environments (unlike 
functional MRI) and can measure spontaneous brain activity in the resting state. Since our recordings were performed in the absence of any stimulus, we can assume that these areas demonstrate continuously increased and decreased changes in activity and connectivity in tinnitus patients (Vanneste et al., 2010).

The main finding is that, depending on the presence of hearing loss, a different kind of tinnitus can be discerned, providing evidence that tinnitus can be associated with bottom-up deafferentation as well as top-down modulation failure (De Ridder et al., $2014 \mathrm{~b}$ ). Based on the pain literature, it has been postulated that tinnitus can be the result of a deficient auditory gating mechanism (Rauschecker et al., 2010, 2015; Leaver et al., 2011). Structural deficits in the ventromedial prefrontal cortex and pregenual anterior cingulate cortex have been associated with a deficient frontostriatal auditory gating mechanism (Rauschecker et al., 2010, 2015; Leaver et al., 2011). This top-down mechanism is a putative central gatekeeper that evaluates the relevance and affective meaning of sensory stimuli and modulates information via descending inhibitory pathways to the thalamic reticular nucleus, which modulates the information flow between the thalamus and the auditory cortex by inhibiting specific thalamic neurons in a highly selective and frequency-specific manner (Yu et al., 2009; Rauschecker et al., 2015). Our findings support this idea by showing that the pregenual anterior cingulate cortex is activated in the alpha frequency band and send information to the auditory cortex in participants who have hearing loss but do not perceive tinnitus. However, in tinnitus patients with and without hearing loss, we observed that the alpha activity is less present but goes together with an increase in theta and gamma activity in the ventromedial prefrontal cortex/pregenual anterior cingulate cortex. An alternative explanation for changes in the pregenual anterior cingulate cortex could be because of tinnitus-related distress. Previous research has already showed that the anterior cingulate cortex is involved in tinnitus-related distress (Vanneste et al., 2010, 2014). Yet, this research showed that distress is related to increased activity in the subgenual anterior cingulate cortex for alpha frequency and the dorsal anterior cingulate cortex for beta frequency. In addition, no correlation was obtained between pregenual anterior cingulate cortex at the theta frequency band and distress.

This top-down gating deficiency for tinnitus patients with normal hearing goes together with a reduced signal transmission to the auditory cortex for the theta frequency band in tinnitus patients. For participants with hearing loss and no tinnitus, this goes together with increased phase coherence between the pregenual anterior cingulate cortex and the auditory cortex for the alpha frequency band. The reduced signal transmission in tinnitus patients with normal hearing goes together with increased activity in the auditory cortex for the theta and gamma frequency bands. For these tinnitus patients, the tinnitus loudness correlates with the activity in the gamma frequency band. These findings support previous findings showing that theta and gamma activity in the auditory cortex are important in tinnitus (Weisz et al., 2005a, 2007a; Lorenz et al., 2009; van der Loo et al., 2009b). Indeed, the link between gamma band activity in the auditory cortex and tinnitus has been identified using both EEG (van der Loo et al., 2009a; De Ridder et al., 2015a) and MEG (Weisz et al., 2005b, 2007b). Our findings further fit with the thalamocortical dysrhythmia model that proposes that normal resting-state alpha activity slows down to theta frequencies in states of deprived input, potentially in the thalamic reticular nucleus because of deficient top-down inhibitory pathways (Llinás et al., 1999, 2005; De Ridder et al., 2011b, 2015b; Vanneste et al., 2018b). This theta activity in the auditory cortex is then coupled to an increase in surrounding gamma activity. Changes of input because of deficient top-down inhibitory pathways can result in a reduction of $\mathrm{GABA}_{\mathrm{A}}$-mediated lateral inhibition, inducing gamma band activity surrounding the deafferented thalamocortical columns (Llinás et al., 2005). In addition, we found phase-amplitude coupling between the theta and gamma frequency bands at the auditory cortex in tinnitus patients with normal hearing. Cross-frequency coupling might be important for integration via low-frequency theta coherence distributed, geographically focal, high-frequency activity (Lisman and Jensen, 2013).

For tinnitus patients with hearing loss, we see increased activity in the parahippocampus and the pregenual anterior cingulate cortex for the theta and gamma frequency bands as well as decreased activity in the auditory cortex. The gamma activity in the parahippocampus further correlates with the loudness of the tinnitus percept. This is in accordance with the recently proposed model for tinnitus that describes a putative multiphase compensation mechanism linking auditory deafferentation to tinnitus (De Ridder et al., 2014a; Vanneste and De Ridder, 2016). The parahippocampus, which is involved in auditory memory, becomes involved in tinnitus with hearing loss (Engelien et al., 2000; De Ridder et al., 2006, 2011a). Similar to tinnitus patients with normal hearing, we observe that coupling between the theta and gamma frequency bands play an important role in the tinnitus percept for tinnitus patients with hearing loss. Different from tinnitus patients with normal hearing, the main generator in tinnitus patients with hearing loss is located in the parahippocampus. This is in accordance with a recent finding that shows a similar pattern of spectral activity to that of thalamocortical dysrhythmia (i.e., theta-gamma) but a different spatial location depending on the disorder (i.e., pain, tinnitus, Parkinson's disease, and depression; Vanneste et al., 2018b). Our data suggest a spectrally equivalent but spatially distinct form of thalamocortical dysrhythmia depending on the deafferentation (top-down vs bottom-up).

Interestingly, for tinnitus patients with hearing loss we also observed a reduced coherence from the pregenual anterior cingulate cortex to the parahippocampus as well as increased activity in pregenual anterior cingulate cortex for the theta frequency band. Although this was not hypothesized, it possible that topdown and bottom-up tinnitus are not mutually exclusive. From a theoretical perspective, both hearing loss (bottom-up) as well as deficient inhibitory mechanisms (top-down) contribute to the tinnitus percept. In a previous study, we have already showed that hearing loss correlates with theta activity in the parahippocampus (Vanneste and De Ridder, 2016). Here, we demonstrate that a top-down gating deficiency can also be added. This fits with our recent finding that the amount of hearing loss and the COMT $\mathrm{Val}^{158} \mathrm{Met}$ polymorphism can increase susceptibility to the clinical manifestation of tinnitus in relationship to the pregenual anterior cingulate cortex (Vanneste et al., 2018a).

Overall, we demonstrate that tinnitus is a network problem that auditory and non-auditory brain areas including the cingulate cortex and the parahippocampus. This is in line with several recent studies using resting state fMRI (Leaver et al., 2016; Chen et al., 2017a,b; Hofmeier et al., 2018). Indeed recent rsfMRI research that showed that the left parahippocampus was active and plays an important hub for tinnitus patients with hearing loss (Maudoux et al., 2012b; Schmidt et al., 2013; Husain et al., 2014). It is important to note that in addition to the areas included in this study (pregenual anterior cingulate cortex, auditory cortex and the parahippocampus), other studies have identified altered 
function in several other parts of the brain including the basal ganglia (Maudoux et al., 2012a), prefrontal cortex (Maudoux et al., 2012a), the limbic system (Chen et al., 2017b; Hofmeier et al., 2018), and the insula (Burton et al., 2012). However, these results have been variable (for review, see Husain and Schmidt, 2014; Elgoyhen et al., 2015) and other studies have found no differences in network processing between tinnitus patients and controls (Wineland et al., 2012; Davies et al., 2014). These findings suggest that tinnitus represents a highly heterogeneous condition (Schecklmann et al., 2012, 2013). Hence, it was suggested that there might be different subtypes of tinnitus. Our research supports this notion of tinnitus subtypes, one driven by top-down mechanisms related to deficient noise-cancelling and another driven by bottom-up mechanisms related to the amount of hearing loss.

A limitation of this study is that we choose a cutoff for separating normal hearing from hearing loss. Other amounts of hearing loss, possibly at a receptor or cellular level, should also be used as cutoff measurements. Furthermore, we only tested hearing acuity in tinnitus patients by means of standard pure tone audiometry limited to $8000 \mathrm{~Hz}$. Recent research has however shown that tinnitus can occur with hearing loss at supraclinical frequencies, i.e., $>8000 \mathrm{~Hz}$ (Melcher et al., 2013). In addition, pure-tone audiometry is not able to detect hidden hearing loss. That is, recent animal research reported that only small effects on audiometric thresholds were observed even with extensive inner hair cell losses, i.e., exceeding 80\% (Lobarinas et al., 2013), and human subjects with tinnitus and a normal audiogram could show changes in wave I of the auditory brainstem response (Schaette and McAlpine, 2011). Future research should include a highfrequency audiogram, auditory brainstem responses, brainstem acoustic reflexes, and speech reception to help further elucidate this problem (Chambers et al., 2016). Another limitation of this study is a lack of subject-specific anatomical forward models. This is sufficient for source reconstruction, but results in more uncertainty in source localization and decreased anatomical precision. Further research should include this and confirm our findings using MRI or PET. An inherent limitation of resting state studies if tinnitus could be influenced by additional factors in addition to tinnitus itself. Next to mood and arousal, attention and internal thought processes during the recording session can affect the results. A possible tinnitus modulations, looking at residual inhibition, spontaneous fluctuations, electromagnetic stimulation of various sorts, lidocaine administration could useful in studying tinnitus correlates, as they allow other types of tinnitus contrasts to be made and to identify the findings that are common to all types of tinnitus study could make the findings more definitive.

In conclusion, our results provide evidence for the existence of a top-down subtype of tinnitus related to a deficient top-down noise-cancelling mechanism alongside a bottom-up subtype of tinnitus that is related to the amount of hearing loss. It was demonstrated that top-down tinnitus is associated with a change in the pregenual anterior cingulate cortex that goes together with increased activity in the auditory cortex. The parahippocampal area becomes involved in tinnitus based on hearing loss. This is in accordance with the idea that tinnitus can have different generators as proposed in a recent model that suggests that different compensation mechanisms at a cortical level can be linked to phantom percepts (Mohan and Vanneste, 2017).

\section{References}

Axelsson A, Ringdahl A (1989) Tinnitus: a study of its prevalence and characteristics. Br J Audiol 23:53-62. CrossRef Medline

Baccalá LA, Sameshima K (2001) Partial directed coherence: a new concept in neural structure determination. Biol Cybern 84:463-474. CrossRef Medline

Barrett AB, Murphy M, Bruno MA, Noirhomme Q, Boly M, Laureys S, Seth AK (2012) Granger causality analysis of steady-state electroencephalographic signals during propofol-induced anaesthesia. PLoS One 7:e29072. CrossRef Medline

Benjamini Y, Hochberg Y (1995) Controlling the false discovery rate: a practical and powerful approach to multiple testing. J R Stat Soc Series B Stat Methodol 57:289-300.

Bloomfield P (2000) Fourier Analysis of Time Series: An Introduction, Ed 2, p 288. New York, NY:John Wiley \& Sons.

Bosman CA, Schoffelen JM, Brunet N, Oostenveld R, Bastos AM, Womelsdorf T, Rubehn B, Stieglitz T, De Weerd P, Fries P (2012) Attentional stimulus selection through selective synchronization between monkey visual areas. Neuron 75:875-888. CrossRef Medline

British Society of Audiology (2008) Recommended procedure: pure tone air and bone conduction threshold audiometry with and without masking and determination of uncomfortable loudness levels. Berkshire, UK.

Burton H, Wineland A, Bhattacharya M, Nicklaus J, Garcia KS, Piccirillo JF (2012) Altered networks in bothersome tinnitus: a functional connectivity study. BMC Neurosci 13:3. CrossRef Medline

Canolty RT, Edwards E, Dalal SS, Soltani M, Nagarajan SS, Kirsch HE, Berger MS, Barbaro NM, Knight RT (2006) High gamma power is phaselocked to theta oscillations in human neocortex. Science 313:1626-1628. CrossRef Medline

Chambers AR, Resnik J, Yuan Y, Whitton JP, Edge AS, Liberman MC, Polley DB (2016) Central gain restores auditory processing following nearcomplete cochlear denervation. Neuron 89:867-879. CrossRef Medline

Chen YC, Xia W, Chen H, Feng Y, Xu JJ, Gu JP, Salvi R, Yin X (2017a) Tinnitus distress is linked to enhanced resting-state functional connectivity from the limbic system to the auditory cortex. Hum Brain Mapp 38:2384-2397. CrossRef Medline

Chen YC, Bo F, Xia W, Liu S, Wang P, Su W, Xu JJ, Xiong Z, Yin X (2017b) Amygdala functional disconnection with the prefrontal-cingulatetemporal circuit in chronic tinnitus patients with depressive mood. Prog Neuropsychopharmacol Biol Psychiatry 79:249-257. CrossRef Medline

Clark JG (1981) Uses and abuses of hearing loss classification. ASHA 23: 493-500. Medline

Congedo M (2002) EureKa! (version 3.0) [Computer Software]. Knoxville, TN: NovaTech EEG.

Congedo M, John RE, De Ridder D, Prichep L, Isenhart R (2010) On the "dependence" of "independent" group EEG sources: an EEG study on two large databases. Brain Topogr 23:134-138. CrossRef Medline

Davies J, Gander PE, Andrews M, Hall DA (2014) Auditory network connectivity in tinnitus patients: a resting-state fMRI study. Int J Audiol 53:192-198. CrossRef Medline

De Ridder D, Fransen H, Francois O, Sunaert S, Kovacs S, Van De Heyning P (2006) Amygdalohippocampal involvement in tinnitus and auditory memory. Acta Otolaryngol Suppl 126:50-53. CrossRef Medline

De Ridder D, Elgoyhen AB, Romo R, Langguth B (2011a) Phantom percepts: tinnitus and pain as persisting aversive memory networks. Proc Natl Acad Sci U S A 108:8075-8080. CrossRef Medline

De Ridder D, van der Loo E, Vanneste S, Gais S, Plazier M, Kovacs S, Sunaert S, Menovsky T, van de Heyning P (2011b) Theta-gamma dysrhythmia and auditory phantom perception. J Neurosurg 114:912-921. CrossRef Medline

De Ridder D, Vanneste S, Freeman W (2014a) The bayesian brain: phantom percepts resolve sensory uncertainty. Neurosci Biobehav Rev 44:4-15. CrossRef Medline

De Ridder D, Vanneste S, Weisz N, Londero A, Schlee W, Elgoyhen AB, Langguth B (2014b) An integrative model of auditory phantom perception: tinnitus as a unified percept of interacting separable subnetworks. Neurosci Biobehav Rev 44:16-32. CrossRef Medline

De Ridder D, Congedo M, Vanneste S (2015a) The neural correlates of subjectively perceived and passively matched loudness perception in auditory phantom perception. Brain Behavior 5:e00331. CrossRef Medline

De Ridder D, Vanneste S, Langguth B, Llinas R (2015b) Thalamocortical 
dysrhythmia: a theoretical update in tinnitus. Front Neurol 6:124. CrossRef Medline

Elgoyhen AB, Langguth B, De Ridder D, Vanneste S (2015) Tinnitus: perspectives from human neuroimaging. Nat Rev Neurosci 16:632-642. CrossRef Medline

Engelien A, Stern E, Isenberg N, Engelien W, Frith C, Silbersweig D (2000) The parahippocampal region and auditory-mnemonic processing. Ann N Y Acad Sci 911:477-485. Medline

Friston K, Moran R, Seth AK (2013) Analysing connectivity with granger causality and dynamic causal modelling. Curr Opin Neurobiol 23:172178. CrossRef Medline

Fuchs M, Kostner J, Wagner M, Ebersole J (2002) A standardized boundary element method volume conductor model. Clinical Neurophysiology 113:702-712. Medline

Geweke J (1982) Measurement of lineair dependence and feedback between multiple time series. J Am Stat Assoc 77:304. CrossRef

Granger CWJ (1969) Investigating causal relations by econometrics models and crosss-spectral methods. Econometrica 37:424. CrossRef

Granger R, Boussinesq J, Girard JP, Rossi JC, Vidal JP (1969) Acid catalyzed oxidation of cyclanones: 3 . Influence of the structure of alkoylcyclohexanones and alkoylcyclopentanones on the orientation of oxidation by $\mathrm{H} 2 \mathrm{O} 2$ and $\mathrm{SeO} 2$ [in French]. Bulletin de la Societe chimique de France 8:2806-2812. Medline

Hiller W, Goebel G (1992) A psychometric study of complaints in chronic tinnitus. J Psychosom Res 36:337-348. CrossRef Medline

Hiller W, Goebel G, Rief W (1994) Reliability of self-rated tinnitus distress and association with psychological symptom patterns. Br J Clin Psychol 33:231-239. CrossRef Medline

Hofmeier B, Wolpert S, Aldamer ES, Walter M, Thiericke J, Braun C, Zelle D, Rüttiger L, Klose U, Knipper M (2018) Reduced sound-evoked and resting-state BOLD fMRI connectivity in tinnitus. Neuroimage Clin 20: 637-649. CrossRef Medline

Holm S (1979) A simple sequential rejective multiple test procedure. Scandinavian J Stat 6:65-70.

Husain FT, Schmidt SA (2014) Using resting state functional connectivity to unravel networks of tinnitus. Hear Res 307:153-162. CrossRef Medline

Husain FT, Carpenter-Thompson JR, Schmidt SA (2014) The effect of mild-to-moderate hearing loss on auditory and emotion processing networks. Front Syst Neurosci 8:10. CrossRef Medline

Jurcak V, Tsuzuki D, Dan I (2007) 10/20, 10/10, and 10/5 systems revisited: their validity as relative head-surface-based positioning systems. Neuroimage 34:1600-1611. CrossRef Medline

Lancaster JL, Woldorff MG, Parsons LM, Liotti M, Freitas CS, Rainey L, Rainey L, Kochunov PV, Nickerson D, Mikiten SA, Fox PT (2000) Automated Talairach Atlas labels for functional brain mapping. Human Brain Mapping, 10, 120-131. Medline

Leaver AM, Renier L, Chevillet MA, Morgan S, Kim HJ, Rauschecker JP (2011) Dysregulation of limbic and auditory networks in tinnitus. Neuron 69:33-43. CrossRef Medline

Leaver AM, Turesky TK, Seydell-Greenwald A, Morgan S, Kim HJ, Rauschecker JP (2016) Intrinsic network activity in tinnitus investigated using functional MRI. Hum Brain Mapp 37:2717-2735. CrossRef Medline

Lisman JE, Jensen O (2013) The theta-gamma neural code. Neuron 77: 1002-1016. CrossRef Medline

Llinás RR, Ribary U, Jeanmonod D, Kronberg E, Mitra PP (1999) Thalamocortical dysrhythmia: a neurological and neuropsychiatric syndrome characterized by magnetoencephalography. Proc Natl Acad Sci U S A 96: 15222-15227. CrossRef Medline

Llinás R, Urbano FJ, Leznik E, Ramírez RR, van Marle HJ (2005) Rhythmic and dysrhythmic thalamocortical dynamics: GABA systems and the edge effect. Trends Neurosci 28:325-333. CrossRef Medline

Lobarinas E, Salvi R, Ding D (2013) Insensitivity of the audiogram to carboplatin induced inner hair cell loss in chinchillas. Hear Res 302:113-120. CrossRef Medline

Logan JM, Sanders AL, Snyder AZ, Morris JC, Buckner RL (2002) Underrecruitment and nonselective recruitment: dissociable neural mechanisms associated with aging. Neuron 33:827-840. CrossRef Medline

Lorenz I, Müller N, Schlee W, Hartmann T, Weisz N (2009) Loss of alpha power is related to increased gamma synchronization: a marker of reduced inhibition in tinnitus? Neurosci Lett 453:225-228. CrossRef Medline

Maudoux A, Lefebvre P, Cabay JE, Demertzi A, Vanhaudenhuyse A, Laureys
S, Soddu A (2012a) Auditory resting-state network connectivity in tinnitus: a functional MRI study. PLoS One 7:e36222. CrossRef Medline

Maudoux A, Lefebvre P, Cabay JE, Demertzi A, Vanhaudenhuyse A, Laureys S, Soddu A (2012b) Connectivity graph analysis of the auditory resting state network in tinnitus. Brain Res 1485:10-21. CrossRef Medline

McCombe A, Baguley D, Coles R, McKenna L, McKinney C, Windle-Taylor P (2001) Guidelines for the grading of tinnitus severity: the results of a working group commissioned by the British Association of Otolaryngologists, Head and Neck Surgeons, 1999. Clin Otolaryngol Allied Sci 26: 388-393. CrossRef Medline

Meeus O, Heyndrickx K, Lambrechts P, De Ridder D, Van de Heyning P (2010) Phase-shift treatment for tinnitus of cochlear origin. Eur Arch Otorhinolaryngol 267:881-888. CrossRef Medline

Meeus O, De Ridder D, Van de Heyning P (2011) Administration of the combination clonazepam-deanxit as treatment for tinnitus. Otol Neurotol 32:701-709. CrossRef Medline

Melcher JR, Knudson IM, Levine RA (2013) Subcallosal brain structure: correlation with hearing threshold at supra-clinical frequencies $(>8$ $\mathrm{kHz}$ ), but not with tinnitus. Hear Res 295:79-86. CrossRef Medline

Moazami-Goudarzi M, Michels L, Weisz N, Jeanmonod D (2010) Temporo-insular enhancement of EEG low and high frequencies in patients with chronic tinnitus: QEEG study of chronic tinnitus patients. BMC Neurosci 11:40. CrossRef Medline

Mohan A, Vanneste S (2017) Adaptive and maladaptive neural compensatory consequences of sensory deprivation-from a phantom percept perspective. Prog Neurobiol 153:1-17. CrossRef Medline

Nichols TE, Holmes AP (2002) Nonparametric permutation tests for functional neuroimaging: a primer with examples. Hum Brain Mapp 15:1-25. CrossRef Medline

Pascual-Marqui R (2007a) Discrete, 3D distributed, linear imaging methods of electric neuronal activity. Part 1: exact, zero error localization. Available at https://arxiv.org/abs/0710.3341.

Pascual-Marqui R (2007b) Instantaneous and lagged measurements of linear and nonlinear dependence between groups of multivariate time series: frequency decomposition. Available at https://arxiv.org/abs/0711.1455.

Pascual-Marqui RD (2002) Standardized low-resolution brain electromagnetic tomography (sLORETA): technical details. Methods Find Exp Clin Pharmacol 24:5-12. Medline

Pascual-Marqui RD, Biscay RJ, Bosch-Bayard J, Lehmann D, Kochi K, Kinoshita T, Yamada N, Sadato N (2014) Assessing direct paths of intracortical causal information flow of oscillatory activity with the isolated effective coherence (iCoh). Front Hum Neurosci 8:448. CrossRef Medline

Pinto PC, Sanchez TG, Tomita S (2010) The impact of gender, age and hearing loss on tinnitus severity. Braz J Otorhinolaryngol 76:18-24. CrossRef Medline

Rauschecker JP, Leaver AM, Mühlau M (2010) Tuning out the noise: limbic-auditory interactions in tinnitus. Neuron 66:819-826. CrossRef Medline

Rauschecker JP, May ES, Maudoux A, Ploner M (2015) Frontostriatal gating of tinnitus and chronic pain. Trends Cogn Sci 19:567-578. CrossRef Medline

Richter P, Werner J, Heerlein A, Kraus A, Sauer H (1998) On the validity of the beck depression inventory: a review. Psychopathology 31:160-168. CrossRef Medline

Schaette R, McAlpine D (2011) Tinnitus with a normal audiogram: physiological evidence for hidden hearing loss and computational model. J Neurosci 31:13452-13457. CrossRef Medline

Schecklmann M, Lehner A, Poeppl TB, Kreuzer PM, Hajak G, Landgrebe M, Langguth B (2012) Cluster analysis for identifying sub-types of tinnitus: a positron emission tomography and voxel-based morphometry study. Brain Res 1485:3-9. CrossRef Medline

Schecklmann M, Lehner A, Poeppl TB, Kreuzer PM, Rupprecht R, Rackl J, Burger J, Frank E, Hajak G, Langguth B, Landgrebe M (2013) Auditory cortex is implicated in tinnitus distress: a voxel-based morphometry study. Brain Struct Funct 218:1061-1070. CrossRef Medline

Schmidt SA, Akrofi K, Carpenter-Thompson JR, Husain FT (2013) Default mode, dorsal attention and auditory resting state networks exhibit differential functional connectivity in tinnitus and hearing loss. PLoS One 8:e76488. CrossRef Medline

Seydel C, Haupt H, Olze H, Szczepek AJ, Mazurek B (2013) Gender and chronic tinnitus: differences in tinnitus-related distress depend on age and duration of tinnitus. Ear Hear 34:661-672. CrossRef Medline 
Siepmann M, Kirch W (2002) Effects of caffeine on topographic quantitative EEG. Neuropsychobiology 45:161-166. CrossRef Medline

Smith TC, Frank E (2016) Introducing machine learning concepts with WEKA. In: Statistical genomics: methods and protocols (Mathe E, Davis S, eds), pp 353-348. New York: Springer.

Stokes PA, Purdon PL (2017) A study of problems encountered in granger causality analysis from a neuroscience perspective. Proc Natl Acad Sci U S A 114:E7063-E7072. CrossRef Medline

Valdes-Sosa PA, Roebroeck A, Daunizeau J, Friston K (2011) Effective connectivity: influence, causality and biophysical modeling. Neuroimage 58 : 339-361. CrossRef Medline

van der Loo E, Gais S, Congedo M, Vanneste S, Plazier M, Menovsky T, Van de Heyning P, De Ridder D (2009a) Tinnitus intensity dependent gamma oscillations of the contralateral auditory cortex. PLoS One 4:e7396. CrossRef Medline

van der Loo E, Gais S, Congedo M, Vanneste S, Plazier M, Menovsky T, Van de Heyning P, De Ridder D (2009b) Tinnitus intensity dependent gamma oscillations of the contralateral auditory cortex. PLoS One 4:e7396. CrossRef

Vanneste S, De Ridder D (2011) Bifrontal transcranial direct current stimulation modulates tinnitus intensity and tinnitus-distress-related brain activity. Eur J Neurosci 34:605-614. CrossRef Medline

Vanneste S, De Ridder D (2016) Deafferentation-based pathophysiological differences in phantom sound: tinnitus with and without hearing loss. Neuroimage 129:80-94. CrossRef Medline

Vanneste S, Plazier M, der Loo Ev, de Heyning PV, Congedo M, De Ridder D (2010) The neural correlates of tinnitus-related distress. Neuroimage 52: 470-480. CrossRef Medline

Vanneste S, Plazier M, van der Loo E, Van de Heyning P, De Ridder D (2011) The difference between uni- and bilateral auditory phantom percept. Clin Neurophysiol 122:578-587. CrossRef Medline

Vanneste S, van Dongen M, De Vree B, Hiseni S, van der Velden E, Strydis C, Joos K, Norena A, Serdijn W, De Ridder D (2013) Does enriched acous- tic environment in humans abolish chronic tinnitus clinically and electrophysiologically? A double blind placebo controlled study. Hear Res 296:141-148. CrossRef Medline

Vanneste S, Congedo M, De Ridder D (2014) Pinpointing a highly specific pathological functional connection that turns phantom sound into distress. Cereb Cortex 24:2268-2282. CrossRef Medline

Vanneste S, Alsalman O, De Ridder D (2018a) COMT and the neurogenetic architecture of hearing loss induced tinnitus. Hear Res 365:1-15. CrossRef Medline

Vanneste S, Song JJ, De Ridder D (2018b) Thalamocortical dysrhythmia detected by machine learning. Nat Commun 9:1103. CrossRef Medline

Volkow ND, Logan J, Fowler JS, Wang GJ, Gur RC, Wong C, Felder C, Gatley SJ, Ding YS, Hitzemann R, Pappas N (2000) Association between agerelated decline in brain dopamine activity and impairment in frontal and cingulate metabolism. Am J Psychiatry 157:75-80. CrossRef Medline

Weisz N, Wienbruch C, Dohrmann K, Elbert T (2005a) Neuromagnetic indicators of auditory cortical reorganization of tinnitus. Brain 128:27222731. CrossRef Medline

Weisz N, Moratti S, Meinzer M, Dohrmann K, Elbert T (2005b) Tinnitus perception and distress is related to abnormal spontaneous brain activity as measured by magnetoencephalography. PLoS Med 2:e153. CrossRef Medline

Weisz N, Dohrmann K, Elbert T (2007a) The relevance of spontaneous activity for the coding of the tinnitus sensation. Prog Brain Res 166:61-70. CrossRef Medline

Weisz N, Müller S, Schlee W, Dohrmann K, Hartmann T, Elbert T (2007b) The neural code of auditory phantom perception. J Neurosci 27:14791484. CrossRef Medline

Wineland AM, Burton H, Piccirillo J (2012) Functional connectivity networks in nonbothersome tinnitus. Otolaryngol Head Neck Surg 147:900906. CrossRef Medline

Yu XJ, Xu XX, He S, He J (2009) Change detection by thalamic reticular neurons. Nat Neurosci 12:1165-1170. CrossRef Medline 